\title{
Breakings of the neutrino $\mu-\tau$ reflection symmetry
}

\section{Zhen-hua Zhao}

Department of Physics, Liaoning Normal University, Dalian 116029, China

E-mail: zhzhao@itp.ac.cn

ABSTRACT: The neutrino $\mu-\tau$ reflection symmetry has been attracting a lot of attention as it predicts the interesting results $\theta_{23}=\pi / 4$ and $\delta= \pm \pi / 2$. But it is reasonable to consider breakings of such a symmetry either from the theoretical considerations or on the basis of experimental results. We thus perform a systematic study for the possible symmetrybreaking patterns and their implications for the mixing parameters. The general treatment is applied to some specific symmetry breaking arising from the renormalization group effects for illustration.

Keywords: Discrete Symmetries, Neutrino Physics, CP violation

ARXIV EPRINT: 1703.04984 


\section{Contents}

1 Introduction 1

2 Breaking of the $\mu-\tau$ reflection symmetry 3

2.1 Numerical results 5

$\begin{array}{lll}2.2 & \text { Analytical approximations } & 12\end{array}$

$\begin{array}{lll}2.3 & \text { RG induced symmetry breaking } & 14\end{array}$

$\begin{array}{lll}3 & \text { Summary } & 18\end{array}$

\section{Introduction}

The discovery of neutrino oscillations indicates that neutrinos are massive and bear flavor mixing. The neutrino mixing arises from the mismatch between their mass and flavor eigenstates, and is described by a $3 \times 3$ unitary matrix $U=U_{l}^{\dagger} U_{\nu}$ (with $U_{l}$ and $U_{\nu}$ being respectively the unitary matrix for diagonalizing the charged-lepton mass matrix $M_{l} M_{l}^{\dagger}$ and neutrino mass matrix $M_{\nu}$ ). In the standard parametrization, $U$ reads

$$
U=P_{\phi}\left(\begin{array}{ccc}
c_{12} c_{13} & s_{12} c_{13} & s_{13} e^{-\mathrm{i} \delta} \\
-s_{12} c_{23}-c_{12} s_{23} s_{13} e^{\mathrm{i} \delta} & c_{12} c_{23}-s_{12} s_{23} s_{13} e^{\mathrm{i} \delta} & s_{23} c_{13} \\
s_{12} s_{23}-c_{12} c_{23} s_{13} e^{\mathrm{i} \delta} & -c_{12} s_{23}-s_{12} c_{23} s_{13} e^{\mathrm{i} \delta} & c_{23} c_{13}
\end{array}\right) P_{\nu}
$$

where $\theta_{i j}$ (for $\left.i j=12,13,23\right)$ are the mixing angles ( with $c_{i j}=\cos \theta_{i j}$ and $s_{i j}=\sin \theta_{i j}$ ) and $\delta$ is the Dirac CP phase. $P_{\nu}=\operatorname{Diag}\left(e^{\mathrm{i} \rho}, e^{\mathrm{i} \sigma}, 1\right)$ contains two Majorana CP phases $\rho$ and $\sigma$, while $P_{\phi}=\operatorname{Diag}\left(e^{\mathrm{i} \phi_{1}}, e^{\mathrm{i} \phi_{2}}, e^{\mathrm{i} \phi_{3}}\right)$ consists of three unphysical phases $\phi_{1,2,3}$ that can be removed via the charged-lepton field rephasing. In addition, neutrino oscillations are also controlled by two mass-squared differences $\Delta m_{i j}^{2}=m_{i}^{2}-m_{j}^{2}$ (for $i j=21,31$ ). Thanks to various neutrino-oscillation experiments [1], the neutrino mixing parameters have been measured to a good accuracy. A global-fit result [2] for them is given by

$$
\begin{aligned}
& \sin ^{2} \theta_{12}=0.308 \pm 0.017, \quad \Delta m_{21}^{2}=(7.54 \pm 0.24) \times 10^{-5} \mathrm{eV}^{2} \text {, } \\
& \sin ^{2} \theta_{13}=0.0234 \pm 0.0020, \quad\left|\Delta m_{31}^{2}\right|=(2.47 \pm 0.06) \times 10^{-3} \mathrm{eV}^{2} \text {. }
\end{aligned}
$$

Note that the sign of $\Delta m_{31}^{2}$ remains undetermined, allowing for two possible neutrino mass orderings $m_{1}<m_{2}<m_{3}$ (referred to as the normal hierarchy and NH for short) or $m_{3}<m_{1}<m_{2}$ (the inverted hierarchy and IH for short). The absolute neutrino mass scale is not known either, but subject to the constraint $m_{1}+m_{2}+m_{3}<0.23 \mathrm{eV}$ from cosmological observations [3]. Particularly noteworthy, a recent result from the NOvA experiment $\left(\theta_{23}=39.5^{\circ} \pm 1.7^{\circ}\right.$ or $52.1^{\circ} \pm 1.7^{\circ}$ in the $\mathrm{NH}$ case $)$ disfavors the popular maximal mixing scenario $\theta_{23}=45^{\circ}$ with $2.6 \sigma$ significance [4]. On the other hand, it is 
interesting to find that the best-fit result for $\delta$ is around $270^{\circ}\left(261^{\circ} \pm 55^{\circ}\right.$ for $\mathrm{NH}$ and $277^{\circ} \pm 43^{\circ}$ for $\left.\mathrm{IH}\right)[5]$.

How to understand the neutrino mixing pattern poses an interesting question. As symmetries (e.g., the $\mathrm{SU}(3)_{\mathrm{q}}$ quark flavor symmetry) have been serving as a guideline for understanding the particle physics, they may play a similar role in addressing the flavor issues. Along this line, many discrete groups have been proposed as the lepton flavor symmetry $[6,7]$. A simplest example is the $\mu-\tau$ permutation symmetry [8-12]: in the basis of $M_{l}$ being diagonal, $M_{\nu}$ should keep unchanged with respect to the transformation $\nu_{\mu} \leftrightarrow \nu_{\tau}$ and thus feature $M_{e \mu}=M_{e \tau}$ and $M_{\mu \mu}=M_{\tau \tau}$ (with $M_{\alpha \beta}$ for $\alpha, \beta=e, \mu, \tau$ being the matrix elements of $M_{\nu}$ ). Such a symmetry (which results in $\theta_{23}=\pi / 4$ and $\theta_{13}=0$ ) was historically motivated by the experimental facts that $\theta_{23}$ takes a value close to $\pi / 4$ while $\theta_{13}$ was only constrained by $\sin ^{2} 2 \theta_{13}<0.18$ [13] (and thus might be negligibly small) at the time. However, the relatively large $\theta_{13} \simeq 0.15$ observed recently [14] requires a significant breaking of this symmetry unless neutrinos are quasi-degenerate in masses [15]. Hence we need to go beyond this simple possibility to accommodate the experimental results in a better way. In this connection, the $\mu-\tau$ reflection symmetry $[12,16]$ may serve as a unique alternative: when $M_{l}$ is diagonal, $M_{\nu}$ should remain invariant under the transformation ${ }^{1}$

$$
\nu_{e} \leftrightarrow \nu_{e}^{c}, \quad \nu_{\mu} \leftrightarrow \nu_{\tau}^{c}, \quad \nu_{\tau} \leftrightarrow \nu_{\mu}^{c}
$$

and thus be characterized by

$$
M_{e \mu}=M_{e \tau}^{*}, \quad M_{\mu \mu}=M_{\tau \tau}^{*}, \quad M_{e e}=M_{e e}^{*}, \quad M_{\mu \tau}=M_{\mu \tau}^{*} .
$$

In addition to allowing for an arbitrary $\theta_{13}$, this symmetry predicts $\theta_{23}=\pi / 4$ and $\delta=$ $\pm \pi / 2$ [19] which are close to the present data, thereby having been attracting a lot of interest [20-34]. Moreover, $\rho$ and $\sigma$ are required to take the trivial values 0 or $\pi / 2$.

Nevertheless, it is hard to believe that the $\mu-\tau$ reflection symmetry can remain as an exact one. On the experimental side, the aforementioned results seem to hint towards $\theta_{23} \neq \pi / 4$ (and possibly $\delta \neq \pm \pi / 2$ ). On the theoretical side, flavor symmetries are generally implemented at a superhigh energy scale and so the renormalization group (RG) running effect may provide a source for the symmetry breaking as we will see. In view of these considerations, it is worthwhile to consider the breaking of this symmetry. In the next section, we perform a systematic study of the possible symmetry-breaking patterns and their implications for the mixing parameters. First of all, we establish an equation set relating the symmetry-breaking parameters in an $M_{\nu}$ of approximate $\mu-\tau$ reflection symmetry and the deviations of mixing parameters from their special values taken in the symmetry context. While the numerical results for these equations are analyzed in section 2.1, some analytical approximations will be derived in section 2.2 to explain the corresponding numerical results. In section 2.3 the general treatment is applied to some specific symmetry breaking arising from the RG running effect. Finally, we summarize our main results in section 3.

\footnotetext{
${ }^{1}$ This operation is a combination of the $\mu-\tau$ exchange and CP conjugate transformations - a typical kind of the generalized CP transformations [17, 18].
} 


\section{Breaking of the $\mu-\tau$ reflection symmetry}

Above all, we define the following parameters to characterize breaking of $\mu-\tau$ reflection symmetry

$$
\epsilon_{1}=\frac{M_{e \mu}-M_{e \tau}^{*}}{M_{e \mu}+M_{e \tau}^{*}}, \quad \epsilon_{2}=\frac{M_{\mu \mu}-M_{\tau \tau}^{*}}{M_{\mu \mu}+M_{\tau \tau}^{*}}, \quad \epsilon_{3}=\frac{\operatorname{Im}\left(M_{e e}\right)}{\operatorname{Re}\left(M_{e e}\right)}, \quad \epsilon_{4}=\frac{\operatorname{Im}\left(M_{\mu \tau}\right)}{\operatorname{Re}\left(M_{\mu \tau}\right)},
$$

by analogy with the discussions about breaking of $\mu-\tau$ permutation symmetry in ref. [35]. Note that they correspond to the four symmetry conditions in eq. (1.4) one by one. These parameters have to be small in magnitude (say $\left|\epsilon_{i}\right| \leq 0.1$ for $i=1,2,3,4$ ) so as to keep the $\mu-\tau$ reflection symmetry as an approximate one. In terms of them, the most general neutrino mass matrix of an approximate $\mu-\tau$ reflection symmetry can always be parameterized in a manner as follows: suppose, at the symmetry level, there is a neutrino mass matrix of the form

$$
M_{\nu}^{(0)}=\left(\begin{array}{ccc}
A_{0} & B_{0} & B_{0}^{*} \\
B_{0} & C_{0} & D_{0} \\
B_{0}^{*} & D_{0} & C_{0}^{*}
\end{array}\right)
$$

in which $A_{0}$ and $D_{0}$ are real. This neutrino mass matrix can be diagonalized by a unitary matrix $U^{(0)}$ (an analogue of $U$ ) with its parameters satisfying the requirements

$$
\phi_{1}^{(0)}=\phi_{2}^{(0)}+\phi_{3}^{(0)}=0, \quad \theta_{23}^{(0)}=\pi / 4, \quad \delta^{(0)}= \pm \pi / 2, \quad \rho^{(0)}, \sigma^{(0)}=0 \text { or } \pi / 2 .
$$

After the symmetry is softly broken, $M_{\nu}^{(0)}$ may receive a general perturbation as given by

$$
M_{\nu}^{(1)}=\left(\begin{array}{lll}
\delta_{e e} & \delta_{e \mu} & \delta_{e \tau} \\
\delta_{e \mu} & \delta_{\mu \mu} & \delta_{\mu \tau} \\
\delta_{e \tau} & \delta_{\mu \tau} & \delta_{\tau \tau}
\end{array}\right)
$$

which can be decomposed into two parts as

$$
M_{\nu}^{(1)}=\frac{1}{2}\left(\begin{array}{ccc}
2 \operatorname{Re}\left(\delta_{e e}\right) & \delta_{e \mu}+\delta_{e \tau}^{*} & \delta_{e \mu}^{*}+\delta_{e \tau} \\
\delta_{e \mu}+\delta_{e \tau}^{*} & \delta_{\mu \mu}+\delta_{\tau \tau}^{*} & 2 \operatorname{Re}\left(\delta_{\mu \tau}\right) \\
\delta_{e \mu}^{*}+\delta_{e \tau} & 2 \operatorname{Re}\left(\delta_{\mu \tau}\right) & \delta_{\mu \mu}^{*}+\delta_{\tau \tau}
\end{array}\right)+\frac{1}{2}\left(\begin{array}{ccc}
2 \mathrm{i} \operatorname{Im}\left(\delta_{e e}\right) & \delta_{e \mu}-\delta_{e \tau}^{*} & \delta_{e \tau}-\delta_{e \mu}^{*} \\
\delta_{e \mu}-\delta_{e \tau}^{*} & \delta_{\mu \mu}-\delta_{\tau \tau}^{*} & 2 \mathrm{i} \operatorname{Im}\left(\delta_{\mu \tau}\right) \\
\delta_{e \tau}-\delta_{e \mu}^{*} & 2 \operatorname{im}\left(\delta_{\mu \tau}\right) & \delta_{\tau \tau}-\delta_{\mu \mu}^{*}
\end{array}\right)
$$

Consequently, the complete neutrino mass matrix $M_{\nu}=M_{\nu}^{(0)}+M_{\nu}^{(1)}$ can be parameterized as

$$
M_{\nu}=\left(\begin{array}{ccc}
A\left(1+\mathrm{i} \epsilon_{3}\right) & B\left(1+\epsilon_{1}\right) & B^{*}\left(1-\epsilon_{1}^{*}\right) \\
B\left(1+\epsilon_{1}\right) & C\left(1+\epsilon_{2}\right) & D\left(1+\mathrm{i} \epsilon_{4}\right) \\
B^{*}\left(1-\epsilon_{1}^{*}\right) & D\left(1+\mathrm{i} \epsilon_{4}\right) & C^{*}\left(1-\epsilon_{2}^{*}\right)
\end{array}\right)
$$

with

$$
\begin{array}{ll}
A=A_{0}+\operatorname{Re}\left(\delta_{e e}\right), & B=B_{0}+\frac{\delta_{e \mu}+\delta_{e \tau}^{*}}{2}, \\
D=D_{0}+\operatorname{Re}\left(\delta_{\mu \tau}\right), & C=C_{0}+\frac{\delta_{\mu \mu}+\delta_{\tau \tau}^{*}}{2},
\end{array}
$$


and

$$
\epsilon_{1}=\frac{\delta_{e \mu}-\delta_{e \tau}^{*}}{2 B}, \quad \epsilon_{2}=\frac{\delta_{\mu \mu}-\delta_{\tau \tau}^{*}}{2 C}, \quad \epsilon_{3}=\frac{\operatorname{Im}\left(\delta_{e e}\right)}{A}, \quad \epsilon_{4}=\frac{\operatorname{Im}\left(\delta_{\mu \tau}\right)}{D} .
$$

It should be noted that $\operatorname{Im}\left(\epsilon_{1,2}\right)$ and $\epsilon_{3,4}$ will transform in a way as

$$
\begin{array}{lll}
\operatorname{Im}\left(\epsilon_{1}\right) \rightarrow \operatorname{Im}\left(\epsilon_{1}\right)+\varphi_{1}+\left(\varphi_{2}+\varphi_{3}\right) / 2, & \epsilon_{3} \rightarrow \epsilon_{3}+2 \varphi_{1}, \\
\operatorname{Im}\left(\epsilon_{2}\right) \rightarrow \operatorname{Im}\left(\epsilon_{2}\right)+\varphi_{2}+\varphi_{3}, & \epsilon_{4} \rightarrow \epsilon_{4}+\varphi_{2}+\varphi_{3},
\end{array}
$$

under the neutrino-field rephasing

$$
\begin{aligned}
& \nu_{e} \rightarrow e^{\mathrm{i} \varphi_{1}} \nu_{e} \simeq\left(1+\mathrm{i} \varphi_{1}\right) \nu_{e}, \\
& \nu_{\tau} \rightarrow e^{\mathrm{i} \varphi_{3}} \nu_{\tau} \simeq\left(1+\mathrm{i} \varphi_{3}\right) \nu_{\tau},
\end{aligned} \quad \nu_{\mu} \rightarrow e^{\mathrm{i} \varphi_{2}} \nu_{\mu} \simeq\left(1+\mathrm{i} \varphi_{2}\right) \nu_{\mu},
$$

with $\varphi_{1,2,3}$ being some small parameters comparable to $\epsilon_{i}$. Taking advantage of such a freedom, one can always achieve $\epsilon_{3,4}=0$ from the general case given by eq. (2.6). In the following discussions, we therefore concentrate on this particular case without loss of generality.

Starting from an $M_{\nu}$ of the form in eq. (2.6) but with $\epsilon_{3,4}=0$, we study the dependence of mixing parameters on $\epsilon_{1,2}$. To this end, we diagonalize such an $M_{\nu}$ with one unitary matrix in a straightforward way

$$
U^{\dagger} M_{\nu} U^{*}=\operatorname{Diag}\left(m_{1}, m_{2}, m_{3}\right) .
$$

The mixing parameters in $U$ are supposed to lie around those special values in eq. (2.3) and the corresponding deviations

$$
\begin{aligned}
& \Delta \phi_{1}=\phi_{1}-0, \quad \Delta \phi=\left(\phi_{2}+\phi_{3}\right) / 2-0, \quad \Delta \theta=\theta_{23}-\pi / 4, \\
& \Delta \delta=\delta-\delta^{(0)}, \quad \Delta \rho=\rho-\rho^{(0)}, \quad \Delta \sigma=\sigma-\sigma^{(0)},
\end{aligned}
$$

are some small quantities. By making perturbation expansions for these small quantities in eq. (2.11), one reaches the following relations connecting the mixing-parameter deviations with $\epsilon_{1,2}$

$$
\begin{aligned}
m_{3} s_{13}^{2} \Delta \delta+\bar{m}_{1} c_{12}^{2} \Delta \rho+\bar{m}_{2} s_{12}^{2} \Delta \sigma & =\left(m_{3} s_{13}^{2}-m_{11}\right) \Delta \phi_{1}, \\
2 m_{12} \bar{s}_{13} \Delta \theta-m_{11} s_{13}^{2} \Delta \delta-\bar{m}_{1} s_{12}^{2} \Delta \rho-\bar{m}_{2} c_{12}^{2} \Delta \sigma & =\left(m_{22}-m_{3}\right) \Delta \phi, \\
{\left[m_{12}+\mathrm{i}\left(m_{11}+m_{3}\right) \bar{s}_{13}\right] \Delta \theta-\left(m_{11}-m_{3}\right) \bar{s}_{13} \Delta \delta } & \\
-2 \bar{m}_{1} c_{12}\left(\mathrm{i} s_{12}+c_{12} \bar{s}_{13}\right) \Delta \rho+2 \bar{m}_{2} s_{12}\left(\mathrm{i} c_{12}-s_{12} \bar{s}_{13}\right) \Delta \sigma & = \\
{\left[m_{12}-\mathrm{i}\left(m_{11}+m_{3}\right) \bar{s}_{13}\right]\left(\mathrm{i} \Delta \phi_{1}+\mathrm{i} \Delta \phi-\epsilon_{1}\right), } & \\
2\left(m_{22}-m_{3}\right) \Delta \theta-2\left(m_{12}-\mathrm{i} m_{11} \bar{s}_{13}\right) \bar{s}_{13} \Delta \delta & \\
-2 \bar{m}_{1} s_{12}\left(\mathrm{i} s_{12}+2 c_{12} \bar{s}_{13}\right) \Delta \rho-2 \bar{m}_{2} c_{12}\left(\mathrm{i} c_{12}-2 s_{12} \bar{s}_{13}\right) \Delta \sigma & = \\
\left(m_{22}+m_{3}-2 \mathrm{i} m_{12} \bar{s}_{13}\right)\left(2 \mathrm{i} \Delta \phi-\epsilon_{2}\right) . &
\end{aligned}
$$


In order to make the expressions compact, the definitions

$$
\begin{aligned}
& m_{11}=\bar{m}_{1} c_{12}^{2}+\bar{m}_{2} s_{12}^{2}, \quad m_{12}=\left(\bar{m}_{1}-\bar{m}_{2}\right) c_{12} s_{12}, \quad m_{22}=\bar{m}_{1} s_{12}^{2}+\bar{m}_{2} c_{12}^{2}, \\
& \bar{m}_{1}=m_{1} \exp \left[2 \mathrm{i} \rho^{(0)}\right], \quad \bar{m}_{2}=m_{2} \exp \left[2 \mathrm{i} \sigma^{(0)}\right], \quad \bar{s}_{13}=-\mathrm{i} s_{13} \exp \left[\mathrm{i} \delta^{(0)}\right],
\end{aligned}
$$

have been taken.

\subsection{Numerical results}

By solving eq. (2.13), one can learn how much mixing-parameter deviations are predicted given some small values of symmetry-breaking parameters. As we will see, the resulting mixing-parameter deviations depend on the neutrino mass spectrum and the combinations of $\rho^{(0)}$ and $\sigma^{(0)}$. So they are to be evaluated as functions of the lightest neutrino mass $\left(m_{1}\right.$ for $\mathrm{NH}$ or $m_{3}$ for $\mathrm{IH}$ ) for various combinations of $\rho^{(0)}$ and $\sigma^{(0)}$ (i.e., $\left[\rho^{(0)}, \sigma^{(0)}\right]=[0,0]$, $[\pi / 2,0],[0, \pi / 2]$ and $[\pi / 2, \pi / 2])$. As typical examples, the mixing-parameter deviations respectively arising from $R_{1} \equiv \operatorname{Re}\left(\epsilon_{1}\right)=0.1$ (in figure 1), $I_{1} \equiv \operatorname{Im}\left(\epsilon_{1}\right)=0.1$ (in figure 2), $\mathrm{R}_{2} \equiv \operatorname{Re}\left(\epsilon_{2}\right)=0.1$ (in figure 3) and $\mathrm{I}_{2} \equiv \operatorname{Im}\left(\epsilon_{2}\right)=0.1$ (in figure 4) are presented in such a way. Here and in the following, the black, red, green and blue colors are assigned to $\Delta \theta$, $\Delta \delta, \Delta \rho$ and $\Delta \sigma$, respectively. To save space, the absolute value of a mixing-parameter deviation will be represented by a dashed line when it is negative. By contrast, the full line will be used when the mixing-parameter deviations are positive. In consideration of the experimental sensitivity, the region where the mixing-parameter deviations have magnitudes smaller than 0.01 has not been shown. In doing the calculations we have specified $\delta^{(0)}=-\pi / 2$. If it takes the opposite value $\pi / 2$, the mixing-parameter deviations produced by $R_{1}$ or $R_{2}$ will change in the way

$$
\Delta \theta \rightarrow \Delta \theta, \quad \Delta \delta \rightarrow-\Delta \delta, \quad \Delta \rho \rightarrow-\Delta \rho, \quad \Delta \sigma \rightarrow-\Delta \sigma,
$$

while those produced by $\mathrm{I}_{1}$ or $\mathrm{I}_{2}$ will change in the way

$$
\Delta \theta \rightarrow-\Delta \theta, \quad \Delta \delta \rightarrow \Delta \delta, \quad \Delta \rho \rightarrow \Delta \rho, \quad \Delta \sigma \rightarrow \Delta \sigma .
$$

The point is that eq. (2.13) stays invariant with respect to the transformations

$$
\begin{aligned}
& \Delta \theta \rightarrow \Delta \theta, \quad \Delta \delta \rightarrow-\Delta \delta, \quad \Delta \rho \rightarrow-\Delta \rho, \quad \Delta \sigma \rightarrow-\Delta \sigma, \\
& \mathrm{R}_{1} \rightarrow \mathrm{R}_{1}, \quad \mathrm{I}_{1} \rightarrow-\mathrm{I}_{1}, \quad \mathrm{R}_{2} \rightarrow \mathrm{R}_{2}, \quad \mathrm{I}_{2} \rightarrow-\mathrm{I}_{2},
\end{aligned}
$$

combined with $\bar{s}_{13} \rightarrow-\bar{s}_{13}$ as well as $\Delta \phi_{1} \rightarrow-\Delta \phi_{1}$ and $\Delta \phi \rightarrow-\Delta \phi$. For the convenience of readers, from the results in figures 1-4 we take some representative values of $\Delta \theta$ (in table 1 ), $\Delta \delta$ (in table 2), $\Delta \rho$ (in table 3 ) and $\Delta \sigma$ (in table 4 ) at $m_{1}\left(m_{3}\right)=0.001,0.01$ and $0.1 \mathrm{eV}$ in the $\mathrm{NH}(\mathrm{IH})$ case. When a value of the mixing-parameter deviations falls in the range $[-0.01,0]$ or $[0,0.01]$, it will be reported as 0.00 or -0.00 .

There are two immediate remarks for the above results. For the convenience of discussions, we use the contribution of $\mathrm{R}_{1}$ to $\Delta \delta$ as an illustration. But the following discussions apply to the contribution of each symmetry-breaking parameter to each mixing-parameter deviation. (1) The resulting $\Delta \delta$ still can be inferred from the results in figure 1 when 

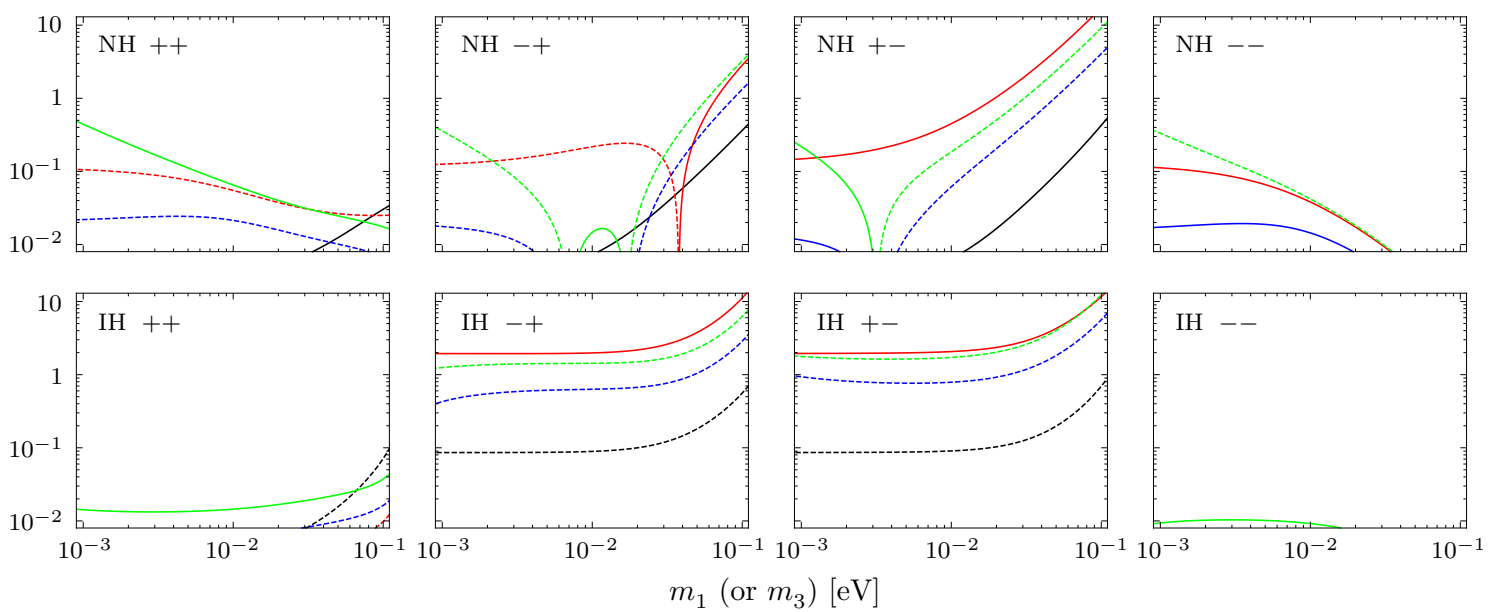

Figure 1. The mixing-parameter deviations induced by $\mathrm{R}_{1}=0.1$ ( $\Delta \theta$ in black, $\Delta \delta$ in red, $\Delta \rho$ in green and $\Delta \sigma$ in blue) against the lightest neutrino mass $m_{1}$ (or $m_{3}$ ) in the NH (or IH) case for various combinations of $\rho^{(0)}$ and $\sigma^{(0)}$ with $\delta^{(0)}=-\pi / 2$. The signs,,++-++- and -- respectively stand for $\left[\rho^{(0)}, \sigma^{(0)}\right]=[0,0],[\pi / 2,0],[0, \pi / 2]$ and $[\pi / 2, \pi / 2]$.
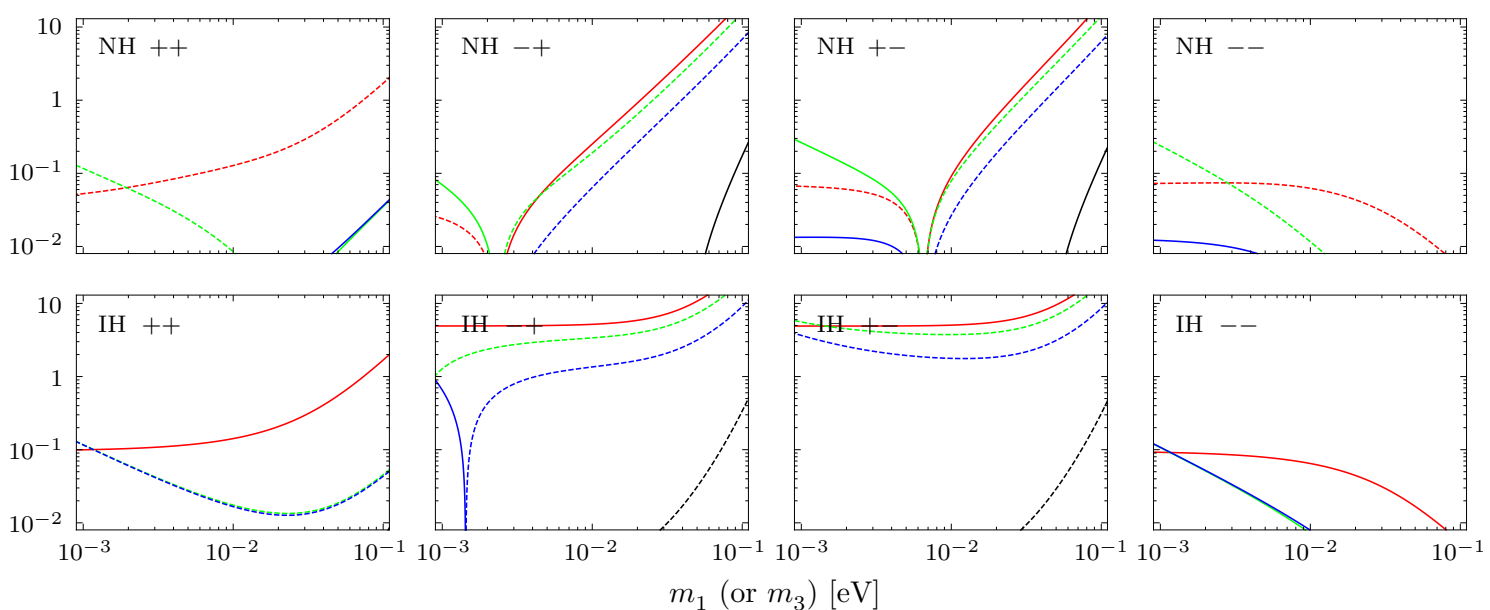

Figure 2. The mixing-parameter deviations induced by $\mathrm{I}_{1}=0.1$ ( $\Delta \theta$ in black, $\Delta \delta$ in red, $\Delta \rho$ in green and $\Delta \sigma$ in blue) against the lightest neutrino mass $m_{1}$ (or $m_{3}$ ) in the $\mathrm{NH}$ (or IH) case for various combinations of $\rho^{(0)}$ and $\sigma^{(0)}$ with $\delta^{(0)}=-\pi / 2$. The signs,,++-++- and -- respectively stand for $\left[\rho^{(0)}, \sigma^{(0)}\right]=[0,0],[\pi / 2,0],[0, \pi / 2]$ and $[\pi / 2, \pi / 2]$.

$\mathrm{R}_{1}$ takes a value other than $0.1 .^{2}$ Since eq. (2.13) is a system of linear equations, $\Delta \delta$ is expected to have a linear dependence on $\mathrm{R}_{1}$, implying that the ratio $\Delta \delta / \mathrm{R}_{1}$ must be a quantity independent of the value of $\mathrm{R}_{1}$. The size of this ratio measures the sensitivity of $\Delta \delta$ to $R_{1}$. When it is of $\leq \mathcal{O}(0.1), \mathcal{O}(1)$ or $\geq \mathcal{O}(10)$, we say that $\Delta \delta$ may receive a small, modest or large contribution from $\mathrm{R}_{1}$. For $\mathrm{R}_{1}=0.1$, the "small", "modest" and "large" contributions are respectively of $\leq \mathcal{O}(0.01), \mathcal{O}(0.1)$ and $\mathcal{O}(1)$. Provided that the

\footnotetext{
${ }^{2}$ Note that in some scenarios (e.g., the RG-induced symmetry breaking as will be discussed in section 2.3) the symmetry-breaking parameters have no chance to acquire a size up to 0.1 .
} 

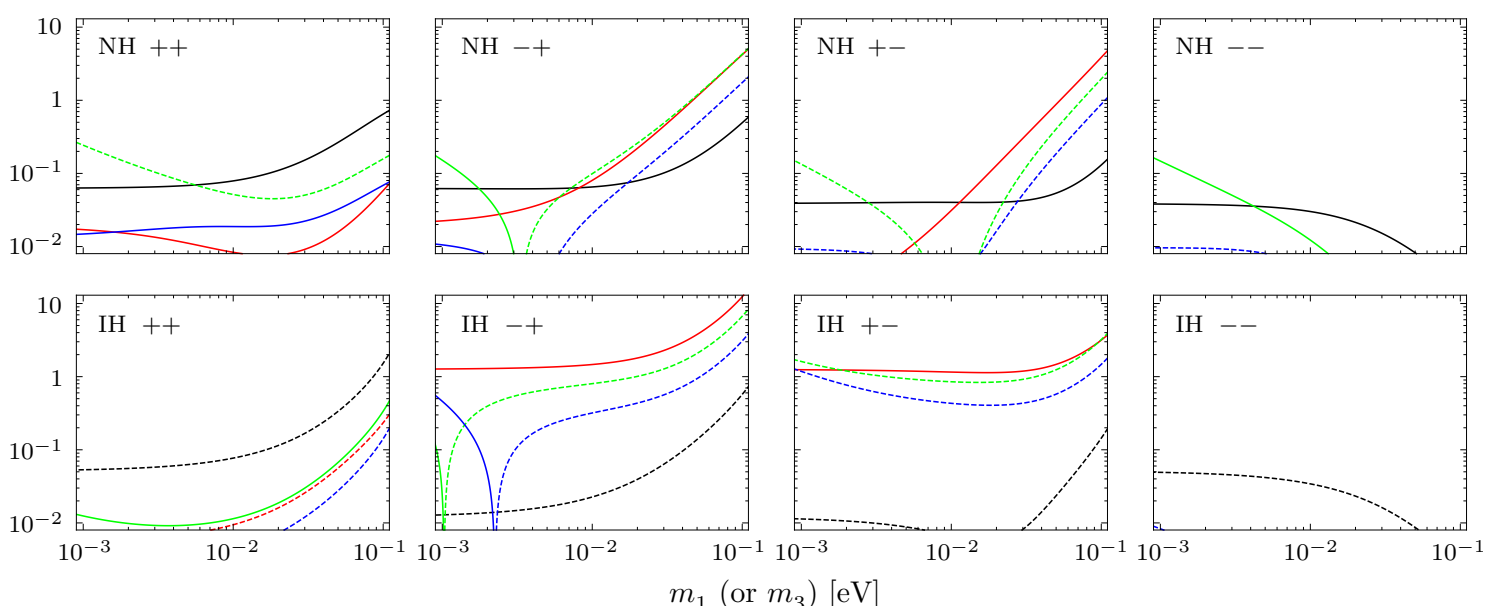

Figure 3. The mixing-parameter deviations induced by $\mathrm{R}_{2}=0.1$ ( $\Delta \theta$ in black, $\Delta \delta$ in red, $\Delta \rho$ in green and $\Delta \sigma$ in blue) against the lightest neutrino mass $m_{1}$ (or $m_{3}$ ) in the NH (or IH) case for various combinations of $\rho^{(0)}$ and $\sigma^{(0)}$ with $\delta^{(0)}=-\pi / 2$. The signs,,++-++- and -- respectively stand for $\left[\rho^{(0)}, \sigma^{(0)}\right]=[0,0],[\pi / 2,0],[0, \pi / 2]$ and $[\pi / 2, \pi / 2]$.
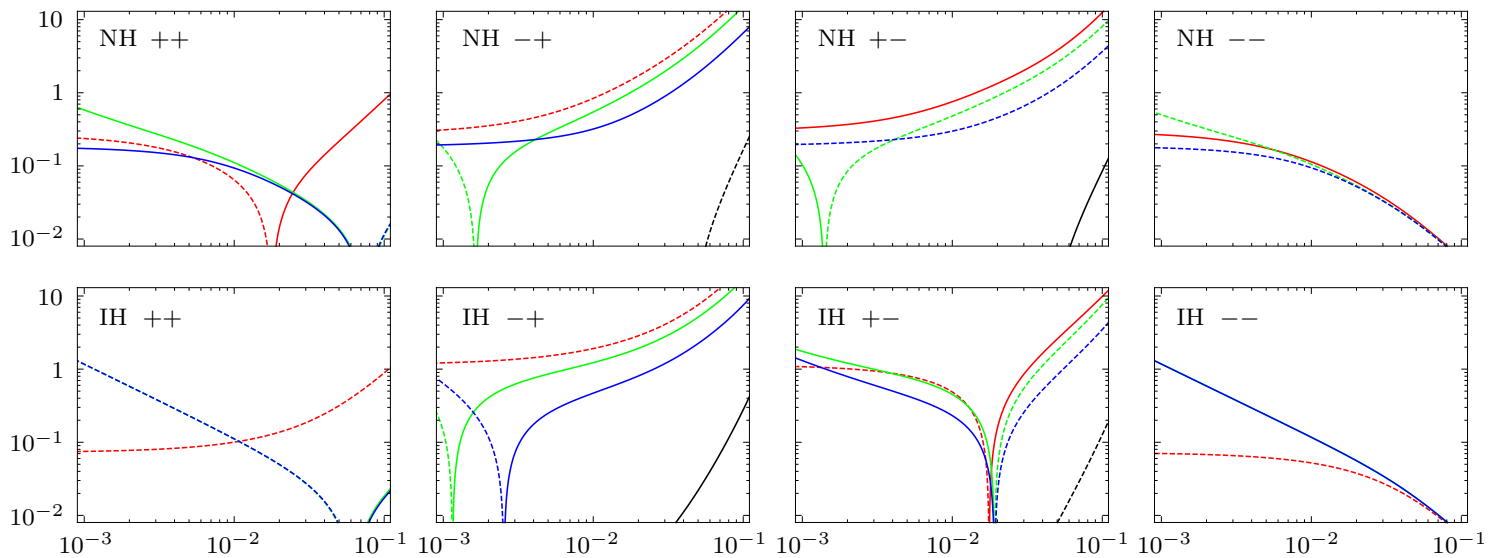

$m_{1}\left(\right.$ or $\left.m_{3}\right)[\mathrm{eV}]$

Figure 4. The mixing-parameter deviations induced by $\mathrm{I}_{2}=0.1$ ( $\Delta \theta$ in black, $\Delta \delta$ in red, $\Delta \rho$ in green and $\Delta \sigma$ in blue) against the lightest neutrino mass $m_{1}$ (or $m_{3}$ ) in the $\mathrm{NH}$ (or IH) case for various combinations of $\rho^{(0)}$ and $\sigma^{(0)}$ with $\delta^{(0)}=-\pi / 2$. The signs,,++-++- and -- respectively stand for $\left[\rho^{(0)}, \sigma^{(0)}\right]=[0,0],[\pi / 2,0],[0, \pi / 2]$ and $[\pi / 2, \pi / 2]$.

$\Delta \delta^{\prime}$ arising from a given value $\mathrm{R}_{1}^{\prime}$ of $\mathrm{R}_{1}$ (e.g., 0.1) has already been known (e.g., the red lines in figure 1 ), the $\Delta \delta^{\prime \prime}$ generated by another value $\mathrm{R}_{1}^{\prime \prime}$ of $\mathrm{R}_{1}$ can be directly obtained as

$$
\Delta \delta^{\prime \prime}=\frac{\Delta \delta^{\prime}}{\mathrm{R}_{1}^{\prime}} \mathrm{R}_{1}^{\prime \prime}=\frac{\mathrm{R}_{1}^{\prime \prime}}{\mathrm{R}_{1}^{\prime}} \Delta \delta^{\prime}
$$

For example, the $\Delta \delta$ produced by $\mathrm{R}_{1}=0.01$ will be a tenth of the result given by figure 1 . (2) But there is one thing to take care of: in some cases (in particular the case of quasidegenerate neutrino mass spectrum combined with $\left.\rho^{(0)} \neq \sigma^{(0)}\right) \Delta \delta$ seems to have gained a contribution of $\geq \mathcal{O}(1)$ from $R_{1}=0.1$. For instance, as shown by the red line of the 


\begin{tabular}{|c|c|c|c|c|}
\hline$\left[\rho^{(0)}, \sigma^{(0)}\right]$ & & $0.001 \mathrm{eV}$ & $0.01 \mathrm{eV}$ & $0.1 \mathrm{eV}$ \\
\hline \hline$[0,0]$ & $\mathrm{R}_{1}=0.1$ & $0.00[-0.00]$ & $0.00[-0.00]$ & $0.03[-0.07]$ \\
\hline$[\pi / 2,0]$ & & $0.00[-0.09]$ & $0.01[-0.09]$ & $0.36[-0.56]$ \\
\hline$[0, \pi / 2]$ & & $0.00[-0.09]$ & $0.01[-0.09]$ & $0.43[-0.70]$ \\
\hline$[\pi / 2, \pi / 2]$ & & $0.00[-0.00]$ & $0.00[-0.00]$ & $0.00[-0.00]$ \\
\hline \hline$[0,0]$ & $\mathrm{I}_{1}=0.1$ & $0.00[0.00]$ & $0.00[0.00]$ & $0.00[0.01]$ \\
\hline$[\pi / 2,0]$ & & $0.00[-0.00]$ & $-0.00[-0.00]$ & $0.17[-0.34]$ \\
\hline$[0, \pi / 2]$ & & $0.00[-0.00]$ & $-0.00[-0.00]$ & $0.15[-0.31]$ \\
\hline$[\pi / 2, \pi / 2]$ & & $0.00[0.00]$ & $0.00[0.00]$ & $0.00[0.00]$ \\
\hline \hline$[0,0]$ & $\mathrm{R}_{2}=0.1$ & $0.06[-0.05]$ & $0.08[-0.08]$ & $0.65[-1.5]$ \\
\hline$[\pi / 2,0]$ & & $0.06[-0.01]$ & $0.07[-0.02]$ & $0.49[-0.57]$ \\
\hline$[0, \pi / 2]$ & & $0.04[-0.01]$ & $0.04[-0.01]$ & $0.13[-0.14]$ \\
\hline$[\pi / 2, \pi / 2]$ & & $0.04[-0.05]$ & $0.03[-0.03]$ & $0.00[-0.00]$ \\
\hline \hline$[0,0]$ & $\mathrm{I}_{2}=0.1$ & $0.00[-0.00]$ & $0.00[-0.00]$ & $-0.00[-0.00]$ \\
\hline$[\pi / 2,0]$ & & $0.00[0.00]$ & $0.00[0.00]$ & $-0.17[0.28]$ \\
\hline$[0, \pi / 2]$ & & $-0.00[0.00]$ & $-0.00[0.00]$ & $0.09[-0.13]$ \\
\hline$[\pi / 2, \pi / 2]$ & & $-0.00[-0.00]$ & $-0.00[-0.00]$ & $-0.00[-0.00]$ \\
\hline
\end{tabular}

Table 1. Some representative values of $\Delta \theta$ (induced by $R_{1}=0.1, I_{1}=0.1, R_{2}=0.1$ and $I_{2}=0.1$, respectively) at $m_{1}\left(m_{3}\right)=0.001,0.01$ and $0.1 \mathrm{eV}$ in the $\mathrm{NH}(\mathrm{IH})$ case for various combinations of $\rho^{(0)}$ and $\sigma^{(0)}$ with $\delta^{(0)}=-\pi / 2$.

sub-figure labelled by "NH +- " in figure $1, \Delta \delta$ takes a value of 10 at $m_{1} \simeq 0.077 \mathrm{eV}$ in the case of $\mathrm{NH}$ combined with $\left[\rho^{(0)}, \sigma^{(0)}\right]=[0, \pi / 2]$. This would never be a realistic result but some signal for the breakdown of our approximation method used to derive eq. (2.13). Nevertheless, the ratio $\Delta \delta / \mathrm{R}_{1}$ which takes a value of 100 in this specific case (implying that even a small $\mathrm{R}_{1}=0.001$ can lead to some sizable $\Delta \delta \simeq 0.1=6^{\circ}$ ) is still useful. To keep $\Delta \delta$ within an acceptably small range in such a case (remember that it is right the possible closeness of $\theta_{23}$ and $\delta$ to $\pi / 4$ and $-\pi / 2$ that motivates us to study the $\mu-\tau$ reflection symmetry), $\mathrm{R}_{1}$ should be of $\leq \mathcal{O}(0.001)$. If $\mathrm{R}_{1}$ were unfortunately much greater than 0.001 , the resulting $\Delta \delta$ would be of $\mathcal{O}(1)$. And one has to invoke an exact method (instead of the approximation one adopted here) to obtain the precise value of $\Delta \delta$. But this is beyond the scope of our interest, because it would be insignificant to discuss the $\mu-\tau$ reflection symmetry any more if $\delta$ turned out to be far from $\pm \pi / 2$.

Given small values of symmetry-breaking parameters (i.e., $\mathrm{R}_{1}=0.1, \mathrm{I}_{1}=0.1, \mathrm{R}_{2}=0.1$ and $\mathrm{I}_{2}=0.1$ ), the resulting $\Delta \theta$ appears as:

1. The $|\Delta \theta|$ from $\mathrm{R}_{1}=0.1$ is: negligibly small in the case of $m_{1} \ll m_{2} \ll m_{3}$ (e.g., the particular case of $m_{1}=0.001 \mathrm{eV}$ in table 1 ); negligibly small or 0.1 in the case of $m_{3} \ll m_{1} \simeq m_{2}$ (e.g., the particular case of $m_{3}=0.001 \mathrm{eV}$ in table 1 ) combined with 


\begin{tabular}{|c|c|c|c|c|}
\hline$\left[\rho^{(0)}, \sigma^{(0)}\right]$ & & $0.001 \mathrm{eV}$ & $0.01 \mathrm{eV}$ & $0.1 \mathrm{eV}$ \\
\hline \hline$[0,0]$ & $\mathrm{R}_{1}=0.1$ & $-0.11[-0.00]$ & $-0.06[-0.00]$ & $-0.03[-0.01]$ \\
\hline$[\pi / 2,0]$ & & $-0.13[1.94]$ & $-0.22[1.98]$ & $2.83[11.2]$ \\
\hline$[0, \pi / 2]$ & & $0.15[1.95]$ & $0.45[2.07]$ & $17.3[11.7]$ \\
\hline$[\pi / 2, \pi / 2]$ & & $0.11[-0.00]$ & $0.04[-0.00]$ & $0.00[-0.00]$ \\
\hline \hline$[0,0]$ & $\mathrm{I}_{1}=0.1$ & $-0.05[0.10]$ & $-0.13[0.14]$ & $-1.70[1.68]$ \\
\hline$[\pi / 2,0]$ & & $-0.02[4.92]$ & $0.25[5.18]$ & $22.0[31.5]$ \\
\hline$[0, \pi / 2]$ & & $-0.07[4.90]$ & $0.09[5.05]$ & $21.6[24.3]$ \\
\hline$[\pi / 2, \pi / 2]$ & & $-0.07[0.09]$ & $-0.06[0.07]$ & $-0.01[0.01]$ \\
\hline \hline$[0,0]$ & $\mathrm{R}_{2}=0.1$ & $0.02[-0.01]$ & $0.01[-0.01]$ & $0.06[-0.23]$ \\
\hline$[\pi / 2,0]$ & & $0.02[1.27]$ & $0.08[1.47]$ & $4.20[12.4]$ \\
\hline$[0, \pi / 2]$ & & $0.00[1.24]$ & $0.03[1.16]$ & $3.86[3.16]$ \\
\hline$[\pi / 2, \pi / 2]$ & & $0.00[-0.01]$ & $-0.00[-0.00]$ & $0.00[-0.00]$ \\
\hline \hline$[0,0]$ & $\mathrm{I}_{2}=0.1$ & $-0.24[-0.08]$ & $-0.06[-0.10]$ & $0.80[-0.89]$ \\
\hline$[\pi / 2,0]$ & & $-0.31[-1.22]$ & $-0.84[-1.91]$ & $-21.1[-25.9]$ \\
\hline$[0, \pi / 2]$ & & $0.33[-1.08]$ & $0.75[-0.49]$ & $12.5[9.86]$ \\
\hline$[\pi / 2, \pi / 2]$ & & $0.27[-0.07]$ & $0.11[-0.05]$ & $0.01[-0.01]$ \\
\hline
\end{tabular}

Table 2. Some representative values of $\Delta \delta$ (induced by $\mathrm{R}_{1}=0.1, \mathrm{I}_{1}=0.1, \mathrm{R}_{2}=0.1$ and $\mathrm{I}_{2}=0.1$, respectively) at $m_{1}\left(m_{3}\right)=0.001,0.01$ and $0.1 \mathrm{eV}$ in the $\mathrm{NH}$ (IH) case for various combinations of $\rho^{(0)}$ and $\sigma^{(0)}$ with $\delta^{(0)}=-\pi / 2$.

$\rho^{(0)}=\sigma^{(0)}$ or $\rho^{(0)} \neq \sigma^{(0)} ; 0.03$ or $\mathcal{O}(1)$ or negligibly small in the case of $m_{1} \simeq m_{2} \simeq m_{3}$ (e.g., the particular case of $m_{1}=0.1 \mathrm{eV}$ in table 1 ) combined with $\left[\rho^{(0)}, \sigma^{(0)}\right]=[0,0]$ or $[\pi / 2,0]$ (and $[0, \pi / 2])$ or $[\pi / 2, \pi / 2]$.

2. The $|\Delta \theta|$ from $\mathrm{I}_{1,2}=0.1$ is: around 0.1 in the case of $m_{1} \simeq m_{2} \simeq m_{3}$ combined with $\rho^{(0)} \neq \sigma^{(0)}$; negligibly small in other cases.

3. The $|\Delta \theta|$ from $R_{2}=0.1$ is: 0.05 in the case of $m_{1} \ll m_{2} \ll m_{3} ; 0.05$ or negligibly small in the case of $m_{3} \ll m_{1} \simeq m_{2}$ combined with $\rho^{(0)}=\sigma^{(0)}$ or $\rho^{(0)} \neq \sigma^{(0)} ; \mathcal{O}(1)$ or 0.1 or negligibly small in the case of $m_{1} \simeq m_{2} \simeq m_{3}$ combined with $\left[\rho^{(0)}, \sigma^{(0)}\right]=[0,0]$ (and $[\pi / 2,0]$ ) or $[0, \pi / 2]$ or $[\pi / 2, \pi / 2]$.

On the other hand, the resulting $\Delta \delta$ turns out to be:

1. The $|\Delta \delta|$ from $\mathrm{R}_{1}=0.1$ is: around 0.1 in the case of $m_{1} \ll m_{2} \ll m_{3}$; negligibly small or $\mathcal{O}(1)$ in the case of $m_{3} \ll m_{1} \simeq m_{2}$ combined with $\rho^{(0)}=\sigma^{(0)}$ or $\rho^{(0)} \neq$ $\sigma^{(0)} ; 0.03$ or $\mathcal{O}(1)$ or negligibly small in the case of $m_{1} \simeq m_{2} \simeq m_{3}$ combined with $\left[\rho^{(0)}, \sigma^{(0)}\right]=[0,0]$ or $[\pi / 2,0]$ (and $\left.[0, \pi / 2]\right)$ or $[\pi / 2, \pi / 2]$. 


\begin{tabular}{|c|c|c|c|c|}
\hline$\left[\rho^{(0)}, \sigma^{(0)}\right]$ & & $0.001 \mathrm{eV}$ & $0.01 \mathrm{eV}$ & $0.1 \mathrm{eV}$ \\
\hline \hline$[0,0]$ & $\mathrm{R}_{1}=0.1$ & $0.44[0.01]$ & $0.07[0.01]$ & $0.02[0.04]$ \\
\hline$[\pi / 2,0]$ & & $-0.36[-1.25]$ & $0.02[-1.43]$ & $-3.24[-6.25]$ \\
\hline$[0, \pi / 2]$ & & $0.22[-1.77]$ & $-0.19[-1.72]$ & $-9.14[-12.4]$ \\
\hline$[\pi / 2, \pi / 2]$ & & $-0.33[0.01]$ & $-0.04[0.01]$ & $-0.00[0.00]$ \\
\hline \hline$[0,0]$ & $\mathrm{I}_{1}=0.1$ & $-0.12[-0.12]$ & $-0.01[-0.02]$ & $0.04[-0.05]$ \\
\hline$[\pi / 2,0]$ & & $0.07[-1.27]$ & $-0.19[-3.38]$ & $-15.7[-20.7]$ \\
\hline$[0, \pi / 2]$ & & $0.26[-5.57]$ & $-0.08[-3.75]$ & $-14.1[-19.0]$ \\
\hline$[\pi / 2, \pi / 2]$ & & $-0.24[0.11]$ & $-0.01[0.01]$ & $-0.00[0.00]$ \\
\hline \hline$[0,0]$ & $\mathrm{R}_{2}=0.1$ & $-0.24[0.01]$ & $-0.05[0.01]$ & $-0.16[0.34]$ \\
\hline$[\pi / 2,0]$ & & $0.15[0.02]$ & $-0.10[-0.80]$ & $-4.27[-6.70]$ \\
\hline$[0, \pi / 2]$ & & $-0.13[-1.61]$ & $-0.00[-0.85]$ & $-1.96[-3.21]$ \\
\hline$[\pi / 2, \pi / 2]$ & & $0.15[-0.00]$ & $0.01[0.00]$ & $0.00[0.00]$ \\
\hline \hline$[0,0]$ & $\mathrm{I}_{2}=0.1$ & $0.58[-1.17]$ & $0.11[-0.11]$ & $-0.01[0.02]$ \\
\hline$[\pi / 2,0]$ & & $-0.18[-0.14]$ & $0.55[1.23]$ & $15.0[17.0]$ \\
\hline$[0, \pi / 2]$ & & $0.10[1.75]$ & $-0.48[0.45]$ & $-8.07[-7.62]$ \\
\hline$[\pi / 2, \pi / 2]$ & & $-0.50[1.17]$ & $-0.11[0.12]$ & $-0.01[0.01]$ \\
\hline
\end{tabular}

Table 3. Some representative values of $\Delta \rho$ (induced by $\mathrm{R}_{1}=0.1, \mathrm{I}_{1}=0.1, \mathrm{R}_{2}=0.1$ and $\mathrm{I}_{2}=0.1$, respectively) at $m_{1}\left(m_{3}\right)=0.001,0.01$ and $0.1 \mathrm{eV}$ in the $\mathrm{NH}(\mathrm{IH})$ case for various combinations of $\rho^{(0)}$ and $\sigma^{(0)}$ with $\delta^{(0)}=-\pi / 2$.

2. The $|\Delta \delta|$ from $\mathrm{I}_{1,2}=0.1$ is: $\mathcal{O}(0.1)$ in the case of $m_{1} \ll m_{2} \ll m_{3} ; 0.1$ or $\mathcal{O}(1)$ in the case of $m_{3} \ll m_{1} \simeq m_{2}$ combined with $\rho^{(0)}=\sigma^{(0)}$ or $\rho^{(0)} \neq \sigma^{(0)} ; \mathcal{O}(1)$ in the case of $m_{1} \simeq m_{2} \simeq m_{3}$ but negligibly small for $\left[\rho^{(0)}, \sigma^{(0)}\right]=[\pi / 2, \pi / 2]$.

3. The $|\Delta \delta|$ from $R_{2}=0.1$ is: negligibly small in the case of $m_{1} \ll m_{2} \ll m_{3}$; negligibly small or $\mathcal{O}(1)$ in the case of $m_{3} \ll m_{1} \simeq m_{2}$ combined with $\rho^{(0)}=\sigma^{(0)}$ or $\rho^{(0)} \neq$ $\sigma^{(0)} ; 0.06$ or $\mathcal{O}(1)$ or negligibly small in the case of $m_{1} \simeq m_{2} \simeq m_{3}$ combined with $\left[\rho^{(0)}, \sigma^{(0)}\right]=[0,0]$ or $[\pi / 2,0]$ (and $\left.[0, \pi / 2]\right)$ or $[\pi / 2, \pi / 2]$.

Note that in the cases where some mixing-parameter deviation acquires a size of $\mathcal{O}(1)$ from $\mathrm{R}_{1}=0.1, \mathrm{I}_{1}=0.1, \mathrm{R}_{2}=0.1$ or $\mathrm{I}_{2}=0.1$, the corresponding symmetry-breaking parameter should be of $\leq \mathcal{O}(0.01)$ to keep this mixing-parameter deviation within an acceptably small range. From the above results one can draw the following observations: (1) The mixing-parameter deviations tend to get remarkably enhanced (or suppressed) in the case of $m_{1} \simeq m_{2} \simeq m_{3}$ combined with $\rho^{(0)} \neq \sigma^{(0)}$ (or $\left.\left[\rho^{(0)}, \sigma^{(0)}\right]=[\pi / 2, \pi / 2]\right)$. (2) When the absolute neutrino mass scale is small and $\rho^{(0)} \neq \sigma^{(0)}$, the mixing-parameter deviations generally have a much greater magnitude in the IH case than in the NH case. (3) $\Delta \theta$ is 


\begin{tabular}{|c|c|c|c|c|}
\hline$\left[\rho^{(0)}, \sigma^{(0)}\right]$ & & $0.001 \mathrm{eV}$ & $0.01 \mathrm{eV}$ & $0.1 \mathrm{eV}$ \\
\hline \hline$[0,0]$ & $\mathrm{R}_{1}=0.1$ & $-0.02[-0.00]$ & $-0.02[-0.01]$ & $-0.01[-0.02]$ \\
\hline$[\pi / 2,0]$ & & $-0.02[-0.43]$ & $0.01[-0.63]$ & $-1.33[-2.88]$ \\
\hline$[0, \pi / 2]$ & & $0.01[-0.94]$ & $-0.06[-0.79]$ & $-4.07[-5.63]$ \\
\hline$[\pi / 2, \pi / 2]$ & & $0.02[-0.01]$ & $0.01[-0.00]$ & $0.00[-0.00]$ \\
\hline \hline$[0,0]$ & $\mathrm{I}_{1}=0.1$ & $0.01[-0.12]$ & $0.00[-0.02]$ & $0.04[-0.04]$ \\
\hline$[\pi / 2,0]$ & & $0.00[0.65]$ & $-0.06[-1.35]$ & $-6.83[-9.09]$ \\
\hline$[0, \pi / 2]$ & & $0.01[-3.65]$ & $-0.03[-1.77]$ & $-6.32[-8.58]$ \\
\hline$[\pi / 2, \pi / 2]$ & & $0.01[0.11]$ & $0.00[0.01]$ & $-0.00[0.00]$ \\
\hline \hline$[0,0]$ & $\mathrm{R}_{2}=0.1$ & $0.02[0.00]$ & $0.02[-0.00]$ & $0.07[-0.14]$ \\
\hline$[\pi / 2,0]$ & & $0.01[0.46]$ & $-0.03[-0.32]$ & $-1.74[-3.08]$ \\
\hline$[0, \pi / 2]$ & & $-0.01[-1.18]$ & $-0.00[-0.43]$ & $-0.88[-1.47]$ \\
\hline$[\pi / 2, \pi / 2]$ & & $-0.01[-0.01]$ & $-0.01[-0.00]$ & $-0.00[-0.00]$ \\
\hline \hline$[0,0]$ & $\mathrm{I}_{2}=0.1$ & $0.17[-1.17]$ & $0.09[-0.11]$ & $-0.01[0.02]$ \\
\hline$[\pi / 2,0]$ & & $0.19[-0.64]$ & $0.32[0.47]$ & $6.57[7.50]$ \\
\hline$[0, \pi / 2]$ & & $-0.20[1.31]$ & $-0.30[0.23]$ & $-3.66[-3.48]$ \\
\hline$[\pi / 2, \pi / 2]$ & & $-0.18[1.17]$ & $-0.09[0.12]$ & $-0.01[0.01]$ \\
\hline
\end{tabular}

Table 4. Some representative values of $\Delta \sigma$ (induced by $\mathrm{R}_{1}=0.1, \mathrm{I}_{1}=0.1, \mathrm{R}_{2}=0.1$ and $\mathrm{I}_{2}=0.1$, respectively) at $m_{1}\left(m_{3}\right)=0.001,0.01$ and $0.1 \mathrm{eV}$ in the $\mathrm{NH}(\mathrm{IH})$ case for various combinations of $\rho^{(0)}$ and $\sigma^{(0)}$ with $\delta^{(0)}=-\pi / 2$.

most sensitive to $\mathrm{R}_{2}$ while $\Delta \delta$ to all the symmetry-breaking parameters. In magnitude, $\Delta \delta$ is generally much greater than $\Delta \theta$.

For illustration, we give a toy example to show how to make use of the above results. In this connection, we discuss how the global-fit results $\theta_{23} /^{\circ}=41.6_{-1.2}^{+1.5}$ and $\delta /{ }^{\circ}=261_{-59}^{+51}$ in the $\mathrm{NH}$ case [5] may arise from an approximate $\mu-\tau$ reflection symmetry. (For simplicity, only the best-fit results will be used.) An interesting possibility is that a single $\mathrm{R}_{2}{ }^{3}$ is responsible for the two measured mixing-parameter deviations $\Delta \theta \simeq-3.4^{\circ}$ and $\Delta \delta \simeq-9^{\circ}$. This can be achieved when the $\Delta \delta$ and $\Delta \theta$ generated by $\mathrm{R}_{2}$ happen to fulfill the condition $\Delta \delta / \Delta \theta=\left(-9^{\circ}\right) /\left(-3.4^{\circ}\right) \simeq 2.65$. With the aid of the sub-figure labelled by "NH -+ " in figure 3 , it turns out that this requirement can be satisfied in the case of $\left[\rho^{(0)}, \sigma^{(0)}\right]=$ $[\pi / 2,0]:$ at $m_{1}=0.019 \mathrm{eV}$, the values of $\Delta \theta / \mathrm{R}_{2}$ and $\Delta \delta / \mathrm{R}_{2}\left(\right.$ as well as $\Delta \rho / \mathrm{R}_{2}$ and $\left.\Delta \sigma / \mathrm{R}_{2}\right)$ respectively read 7.8 and 20.7 (as well as -24.5 and -9 ). Accordingly, $R_{2} \simeq-0.076$ is capable of producing $\theta_{23} \simeq 41.6^{\circ}$ and $\delta=261^{\circ}$ (as well as $\rho \simeq 100.7^{\circ}$ and $\sigma \simeq 3.9^{\circ}$ ).

\footnotetext{
${ }^{3}$ Of course, in a realistic context, the mixing-parameter deviations may receive contributions from not merely one symmetry-breaking parameter.
} 

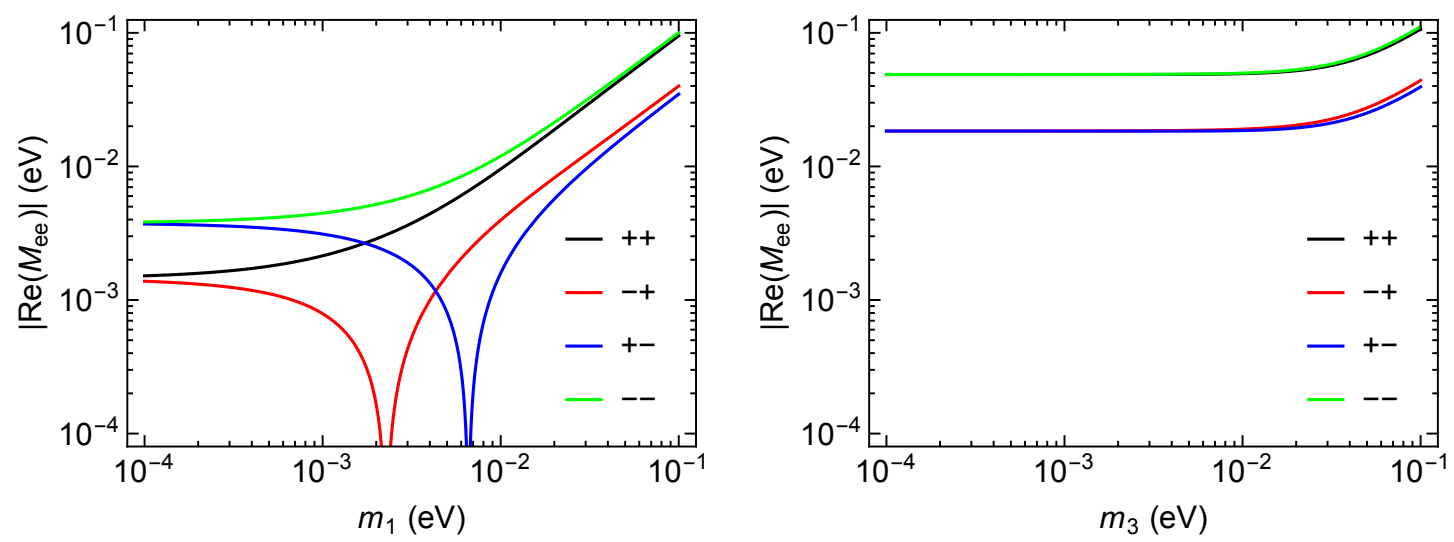

Figure 5. The possible values of $\left|\operatorname{Re}\left(M_{e e}\right)\right|$ against the lightest neutrino mass $m_{1}$ (or $\left.m_{3}\right)$ in the NH (or IH) case for various combinations of $\rho^{(0)}$ and $\sigma^{(0)}$. The signs,,++-++- and -- respectively stand for $\left[\rho^{(0)}, \sigma^{(0)}\right]=[0,0],[\pi / 2,0],[0, \pi / 2]$ and $[\pi / 2, \pi / 2]$.

Finally, we discuss the consequences of breaking of $\mu-\tau$ reflection symmetry on the allowed range of effective Majorana neutrino mass $\left|M_{e e}\right|$ which directly controls the rates of neutrinoless double-beta decays [36-39]. For this purpose, one obtains

$$
\begin{aligned}
& \operatorname{Re}\left(M_{e e}\right) \simeq \bar{m}_{1} c_{12}^{2} c_{13}^{2}+\bar{m}_{2} s_{12}^{2} c_{13}^{2}-m_{3} s_{13}^{2} \\
& \operatorname{Im}\left(M_{e e}\right) \simeq \bar{m}_{1} c_{12}^{2} \Delta \rho+\bar{m}_{2} s_{12}^{2} \Delta \sigma+m_{3} s_{13}^{2} \Delta \delta+\left(m_{11}-m_{3} s_{13}^{2}\right) \Delta \phi_{1} .
\end{aligned}
$$

Because the symmetry-breaking parameter $\epsilon_{3}=\operatorname{Im}\left(M_{e e}\right) / \operatorname{Re}\left(M_{e e}\right)$ should be a small quantity (e.g., $\left|\epsilon_{3}\right| \leq 0.1$ ) if we want to maintain the $\mu-\tau$ reflection symmetry as an approximate one, the value of

$$
\left|M_{e e}\right|=\sqrt{\left[\operatorname{Re}\left(M_{e e}\right)\right]^{2}+\left[\operatorname{Im}\left(M_{e e}\right)\right]^{2}} \simeq\left|\operatorname{Re}\left(M_{e e}\right)\right|\left(1+\frac{\epsilon_{3}^{2}}{2}\right),
$$

can be well approximated by that of $\left|\operatorname{Re}\left(M_{e e}\right)\right|$. It is thus fair to say that the consequences of breaking of $\mu-\tau$ reflection symmetry on the allowed range of $\left|M_{e e}\right|$ are negligibly small. In figure 5 we present the possible values of $\left|\operatorname{Re}\left(M_{e e}\right)\right|$ as a function of the lightest neutrino mass $m_{1}$ (or $m_{3}$ ) in the $\mathrm{NH}$ (or IH) case for various combinations of $\rho^{(0)}$ and $\sigma^{(0)}$ [23]. (1) In the NH case, the three components of $\left|\operatorname{Re}\left(M_{e e}\right)\right|$ add constructively to a maximal level for $\left[\rho^{(0)}, \sigma^{(0)}\right]=[\pi / 2, \pi / 2]$. By contrast, the three components will cancel each other out (i.e., $\left.\left|\operatorname{Re}\left(M_{e e}\right)\right| \simeq 0\right)$ at $m_{1} \simeq 0.002 \mathrm{eV}($ or $0.007 \mathrm{eV})$ for $\left[\rho^{(0)}, \sigma^{(0)}\right]=[\pi / 2,0]($ or $[0, \pi / 2])$. (2) In the IH case, the value of $\left|\operatorname{Re}\left(M_{e e}\right)\right|$ is mainly determined by the first two components as the third one is highly suppressed. Because of $m_{1} \simeq m_{2}$ in the IH case, $\left|\operatorname{Re}\left(M_{e e}\right)\right|$ approximates to $m_{1}\left(\right.$ or $\left.m_{1}\left(c_{12}^{2}-s_{12}^{2}\right)\right)$ for $\rho^{(0)}=\sigma^{(0)}\left(\right.$ or $\left.\rho^{(0)} \neq \sigma^{(0)}\right)$.

\subsection{Analytical approximations}

In this section, we give the analytical expressions of $\Delta \delta$ and $\Delta \theta$ (which are of more practical interest than $\Delta \rho$ and $\Delta \sigma$ since the Majorana phases cannot be pinned down in a foreseeable 
future) to explain the numerical results. After a lengthy but straightforward calculation, one obtains the approximation results

$$
\begin{aligned}
\Delta \delta \simeq & {\left[2 \mathrm{~T}\left(\bar{m}_{1}+\bar{m}_{2}\right)\left(\bar{m}_{1}-m_{3}\right)\left(\bar{m}_{2}-m_{3}\right) \bar{s}_{13}\right]^{-1}\left\{\left[2 \mathrm{~T} m_{12}\left(m_{22}-m_{3}\right)\left(\bar{m}_{1}+\bar{m}_{2}-2 m_{3} s_{13}^{2}\right)\right.\right.} \\
& \left.-4 m_{12}^{2}\left(m_{11}+m_{3}+\mathrm{T} m_{12}\right) s_{13}^{2}+4\left(\bar{m}_{1}-m_{3}\right)\left(\bar{m}_{2}-m_{3}\right)\left(m_{11}+m_{3}\right) s_{13}^{2}\right] \mathrm{R}_{1}-\left[4 m_{12}\right. \\
& \times\left(\bar{m}_{1}-m_{3}\right)\left(\bar{m}_{2}-m_{3}\right)-2 \mathrm{~T}\left(\bar{m}_{1}+\bar{m}_{2}-2 m_{3} s_{13}^{2}\right)\left(m_{11}+m_{3}\right)\left(m_{22}-m_{3}\right)-4 \mathrm{~T} \Omega \\
& \left.\times m_{12}^{2} m_{3} s_{13}^{2}\right] \bar{s}_{13} \mathrm{I}_{1}-\left[\mathrm{T} m_{12}\left(m_{22}+m_{3}\right)\left(\bar{m}_{1}+\bar{m}_{2}-2 m_{3} s_{13}^{2}\right)-2 \mathrm{~T} m_{12}\left(\bar{m}_{1}-m_{3}\right)\right. \\
& \left.\times\left(\bar{m}_{2}-m_{3}\right) s_{13}^{2}-2\left(m_{11}-m_{3}\right)\left(m_{22}+m_{3}\right)\left(m_{11}+m_{3}+\mathrm{T} m_{12}\right) s_{13}^{2}\right] \mathrm{R}_{2} \\
& \left.+\mathrm{T}\left(m_{22}+m_{3}\right)\left[m_{12}^{2}-\left(m_{22}-m_{3}\right)\left(2 m_{11}+m_{22}-m_{3}\right)\right] \bar{s}_{13} \mathrm{I}_{2}\right\}, \\
\Delta \theta \simeq & {\left[2 \mathrm{~T}\left(\bar{m}_{1}+\bar{m}_{2}\right)\left(\bar{m}_{1}-m_{3}\right)\left(\bar{m}_{2}-m_{3}\right)\right]^{-1}\left\{2 \mathrm{~T}\left(\bar{m}_{1}+\bar{m}_{2}\right)\left[m_{12}^{2}-\left(m_{11}^{2}-m_{3}^{2}\right) s_{13}^{2}\right] \mathrm{R}_{1}\right.} \\
& +4 m_{11} m_{12}\left[\mathrm{~T}\left(\bar{m}_{1}+\bar{m}_{2}\right)+2 m_{12} s_{13}^{2}\right] \bar{s}_{13} \mathrm{I}_{1}-\mathrm{T}\left(\bar{m}_{1}+\bar{m}_{2}\right)\left(m_{11}-m_{3}\right)\left(m_{22}+m_{3}\right) \mathrm{R}_{2} \\
& \left.-\mathrm{T} m_{12}\left[\left(\bar{m}_{1}+\bar{m}_{2}\right)\left(2 m_{11}+m_{22}-m_{3}\right)-2 m_{11}\left(m_{22}+m_{3}\right) s_{13}^{2}\right] \bar{s}_{13} \mathrm{I}_{2}\right\},
\end{aligned}
$$

with $\mathrm{T}=\tan 2 \theta_{12}$ and $\Omega=\left(m_{11}+m_{3}\right) /\left(m_{11}-m_{3}\right)$.

For illustration, we discuss the possible values of $\Delta \delta$ and $\Delta \theta$ in several typical cases. (1) In the case of $m_{1} \ll m_{2} \simeq \sqrt{\Delta m_{21}^{2}} \ll m_{3} \simeq \sqrt{\left|\Delta m_{31}^{2}\right|}, \Delta \delta$ and $\Delta \theta$ approximate to

$$
\begin{aligned}
& \Delta \delta \simeq \frac{m_{3}}{2 \bar{m}_{2} \bar{s}_{13}}\left[\left(2 r c_{12} s_{12}+\frac{4}{\mathrm{~T}} s_{13}^{2}\right) \mathrm{R}_{1}-\bar{s}_{13} \mathrm{I}_{2}+\left(r c_{12} s_{12}-\frac{2}{\mathrm{~T}} s_{13}^{2}\right) \mathrm{R}_{2}\right]-2 s_{12}^{2} \mathrm{I}_{1} \\
& \Delta \theta \simeq s_{13}^{2} \mathrm{R}_{1}-2|r| c_{12} s_{12}^{3} \bar{s}_{13} \mathrm{I}_{1}+\frac{1}{2} \mathrm{R}_{2}-\frac{\bar{m}_{2}}{2 m_{3}} c_{12} s_{12} \bar{s}_{13} \mathrm{I}_{2}
\end{aligned}
$$

where $r=\Delta m_{21}^{2} / \Delta m_{31}^{2}$ takes a value of \pm 0.03 for $\mathrm{NH}$ or IH. One finds that $\Delta \delta(\Delta \theta)$ can receive modest contributions from $\mathrm{R}_{1}$ and $\mathrm{I}_{1,2}\left(\mathrm{R}_{2}\right)$. (2) For $m_{1} \simeq m_{2} \simeq \sqrt{\left|\Delta m_{31}^{2}\right|} \gg m_{3}$, in which case one has $m_{2}-m_{1} \simeq \Delta m_{21}^{2} /\left(2 \sqrt{\left|\Delta m_{31}^{2}\right|}\right)$, the results are strongly dependent on the combinations of $\rho^{(0)}$ and $\sigma^{(0)}$. If $\rho^{(0)}$ and $\sigma^{(0)}$ take the same value, $\Delta \delta$ and $\Delta \theta$ are simplified to

$$
\begin{aligned}
& \Delta \delta \simeq \mathrm{I}_{1}-\frac{3}{4} \mathrm{I}_{2}+\frac{1}{4 \mathrm{~T} \bar{s}_{13}}\left[\left(4 s_{13}^{2}-2 \mathrm{~T}|r| c_{12} s_{12}\right) \mathrm{R}_{1}+\left(2 s_{13}^{2}+\mathrm{T}|r| c_{12} s_{12}\right) \mathrm{R}_{2}\right], \\
& \Delta \theta \simeq-s_{13}^{2} \mathrm{R}_{1}-|r| c_{12} s_{12} \bar{s}_{13}\left(\mathrm{I}_{1}-\frac{3}{4} \mathrm{I}_{2}\right)-\frac{1}{2} \mathrm{R}_{2} .
\end{aligned}
$$

It turns out that $\Delta \delta(\Delta \theta)$ can acquire modest contributions from $\mathrm{I}_{1}$ and $\mathrm{I}_{2}\left(\mathrm{R}_{2}\right)$. When $\rho^{(0)}$ and $\sigma^{(0)}$ differ from each other, one will have

$$
\begin{aligned}
& \Delta \delta \simeq \frac{1}{|r|}\left[-32 c_{12}^{3} s_{12}^{3} \bar{s}_{13} \mathrm{R}_{1}+4 \cos 2 \theta_{12} \mathrm{I}_{1}-4 c_{12} s_{12} \bar{s}_{13} \mathrm{R}_{2}-4 c_{12}^{2} s_{12}^{2} \cos 2 \theta_{12} \mathrm{I}_{2}\right], \\
& \Delta \theta \simeq-4 c_{12}^{2} s_{12}^{2} \mathrm{R}_{1}-c_{12} s_{12} \cos 2 \theta_{12}\left[1-\frac{4}{|r|} \cos 2 \theta_{12} s_{13}^{2}\right] \bar{s}_{13}\left(4 \mathrm{I}_{1}-\mathrm{I}_{2}\right)-\frac{1}{2} \cos ^{2} 2 \theta_{12} \mathrm{R}_{2} .
\end{aligned}
$$

Enhanced by the factor $1 /|r|, \Delta \delta$ is easy to gain a large contribution. On the other hand, $\Delta \theta$ can only obtain a modest contribution from $R_{1}$. (3) When it comes to $m_{1} \simeq m_{2} \simeq m_{3} \simeq m_{0}$, in which case $m_{2}-m_{1} \simeq \Delta m_{21}^{2} /\left(2 m_{0}\right)$ and $m_{3}-m_{1} \simeq \Delta m_{31}^{2} /\left(2 m_{0}\right)$, the mixing-parameter 
deviations may get remarkably magnified in some cases. For $\left[\rho^{(0)}, \sigma^{(0)}\right]=[0,0], \Delta \delta$ and $\Delta \theta$ are approximately given by

$$
\begin{aligned}
& \Delta \delta \simeq \frac{1}{\mathrm{~T} \bar{s}_{13}}\left[\left(\operatorname{Trc}_{12} s_{12}+2 s_{13}^{2}\right) \mathrm{R}_{1}+\frac{2 m_{0}^{2}}{\Delta m_{31}^{2}}\left(\operatorname{Trc}_{12} s_{12}-2 s_{13}^{2}\right) \mathrm{R}_{2}\right]-\frac{2 m_{0}^{2}}{\Delta m_{31}^{2}}\left(2 \mathrm{I}_{1}-\mathrm{I}_{2}\right), \\
& \Delta \theta \simeq \frac{2 m_{0}^{2}}{\Delta m_{31}^{2}}\left[2 s_{13}^{2} \mathrm{R}_{1}-r c_{12} s_{12} \bar{s}_{13}\left(2 \mathrm{I}_{1}-\mathrm{I}_{2}\right)+\mathrm{R}_{2}\right] .
\end{aligned}
$$

Because of the enhancement factor $m_{0}^{2} / \Delta m_{31}^{2}, \Delta \delta(\Delta \theta)$ can get large contributions from $\mathrm{I}_{1}$ and $\mathrm{I}_{2}\left(\mathrm{R}_{2}\right)$. In the case of $\left[\rho^{(0)}, \sigma^{(0)}\right]=[\pi / 2,0], \Delta \delta$ and $\Delta \theta$ appear as

$$
\begin{aligned}
& \Delta \delta \simeq \frac{2 m_{0}^{2}}{\Delta m_{21}^{2}}\left\{\left[\frac{4}{\mathrm{~T}} s_{12}^{2}-2 c_{12} s_{12}\left(1+2 s_{12}^{2}\right)\right] \bar{s}_{13} \mathrm{R}_{1}+2 \cos 2 \theta_{12} \mathrm{I}_{1}-2 c_{12} s_{12} \bar{s}_{13} \mathrm{R}_{2}-c_{12}^{2} \mathrm{I}_{2}\right\}, \\
& \Delta \theta \simeq \frac{2 m_{0}^{2}}{\Delta m_{31}^{2}}\left[2 c_{12}^{2} s_{12}^{2} \mathrm{R}_{1}-\frac{4 m_{0}^{2}}{\Delta m_{21}^{2}} c_{12} s_{12} \cos 2 \theta_{12} \bar{s}_{13}^{3}\left(2 \cos 2 \theta_{12} \mathrm{I}_{1}-c_{12}^{2} \mathrm{I}_{2}\right)+c_{12}^{4} \mathrm{R}_{2}\right] .
\end{aligned}
$$

When $\left[\rho^{(0)}, \sigma^{(0)}\right]=[0, \pi / 2]$, the results become

$$
\begin{aligned}
& \Delta \delta \simeq \frac{2 m_{0}^{2}}{\Delta m_{21}^{2}}\left\{-\left[\frac{4}{\mathrm{~T}} c_{12}^{2}+2 c_{12} s_{12}\left(1+2 c_{12}^{2}\right)\right] \bar{s}_{13} \mathrm{R}_{1}+2 \cos 2 \theta_{12} \mathrm{I}_{1}-2 c_{12} s_{12} \bar{s}_{13} \mathrm{R}_{2}+s_{12}^{2} \mathrm{I}_{2}\right\}, \\
& \Delta \theta \simeq \frac{2 m_{0}^{2}}{\Delta m_{31}^{2}}\left[2 c_{12}^{2} s_{12}^{2} \mathrm{R}_{1}-\frac{4 m_{0}^{2}}{\Delta m_{21}^{2}} c_{12} s_{12} \cos 2 \theta_{12} \bar{s}_{13}^{3}\left(2 \cos 2 \theta_{12} \mathrm{I}_{1}+s_{12}^{2} \mathrm{I}_{2}\right)+s_{12}^{4} \mathrm{R}_{2}\right] .
\end{aligned}
$$

In these two cases $\Delta \delta$ is greatly enhanced by the factor $m_{0}^{2} / \Delta m_{21}^{2}$, while the enhancement factor $m_{0}^{2} / \Delta m_{31}^{2}$ for $\Delta \theta$ is also significant but not so huge. Finally, $\left[\rho^{(0)}, \sigma^{(0)}\right]=[\pi / 2, \pi / 2]$ will lead us to

$$
\begin{aligned}
& \Delta \delta \simeq-\frac{\Delta m_{31}^{2}}{4 m_{0}^{2} \bar{s}_{13}}\left[\left(r c_{12} s_{12}+\frac{2}{\mathrm{~T}} s_{13}^{2}\right) \mathrm{R}_{1}+\bar{s}_{13}\left(\mathrm{I}_{1}-\mathrm{I}_{2}\right)\right], \\
& \Delta \theta \simeq \frac{\Delta m_{31}^{2}}{8 m_{0}^{2}}\left[2 s_{13}^{2} \mathrm{R}_{1}-2 r c_{12} s_{12} \bar{s}_{13}\left(\mathrm{I}_{1}-\mathrm{I}_{2}\right)+\mathrm{R}_{2}\right] .
\end{aligned}
$$

It is easy to see that all the mixing-parameter deviations are extremely suppressed in this case. One will find that all the above analytical results agree well with the corresponding numerical results.

\subsection{RG induced symmetry breaking}

This section is devoted to the RG-induced breaking of $\mu-\tau$ reflection symmetry. A flavor symmetry $[6,7]$ together with the associated new fields is usually introduced at an energy scale $\Lambda_{\mathrm{FS}}$ much higher than the electroweak (EW) one $\Lambda_{\mathrm{EW}}$. In this case one must consider the RG running effect when confronting the flavor-symmetry model with the low-energy data [40]. During the RG evolution process the significant difference between $m_{\mu}$ and $m_{\tau}$ can serve as a unique source for the breaking of $\mu-\tau$ reflection symmetry. The general symmetry breaking studied in the above thus finds an interesting application in such a specific situation [41-44]. The energy dependence of neutrino mass matrix is described by its RG equation, which at the one-loop level appears as [45-49]

$$
16 \pi^{2} \frac{\mathrm{d} M_{\nu}}{\mathrm{d} t}=C\left(Y_{l}^{\dagger} Y_{l}\right)^{T} M_{\nu}+C M_{\nu}\left(Y_{l}^{\dagger} Y_{l}\right)+\alpha M_{\nu}
$$


Here $t$ is defined as $\ln \left(\mu / \mu_{0}\right)$ with $\mu$ denoting the renormalization scale, whereas $C$ and $\alpha$ read

$$
\begin{aligned}
& C=-\frac{3}{2}, \quad \alpha \simeq-3 g_{2}^{2}+6 y_{t}^{2}+\lambda, \quad \text { in the SM ; } \\
& C=1, \quad \alpha \simeq-\frac{6}{5} g_{1}^{2}-6 g_{2}^{2}+6 y_{t}^{2}, \quad \text { in the MSSM . }
\end{aligned}
$$

In eq. (2.29) the $\alpha$-term is flavor universal and therefore just contributes an overall rescaling factor (which will be referred to as $I_{\alpha}$ ), while the other two terms may modify the structure of $M_{\nu}$. In the basis under study, the Yukawa coupling matrix of three charged leptons is given by $Y_{l}=\operatorname{Diag}\left(y_{e}, y_{\mu}, y_{\tau}\right)$. In light of $y_{e} \ll y_{\mu} \ll y_{\tau}$, it is reasonable to neglect the contributions of $y_{e}$ and $y_{\mu}$. Integration of the RG equation enables us to connect the neutrino mass matrix $M_{\nu}\left(\Lambda_{\mathrm{FS}}\right)$ at $\Lambda_{\mathrm{FS}}$ with the corresponding one at $\Lambda_{\mathrm{EW}}$ in a manner as $[50,51]$

$$
M_{\nu}\left(\Lambda_{\mathrm{EW}}\right)=I_{\alpha} I_{\tau}^{\dagger} M_{\nu}\left(\Lambda_{\mathrm{FS}}\right) I_{\tau}^{*},
$$

where $I_{\tau} \simeq \operatorname{Diag}\left\{1,1,1-\Delta_{\tau}\right\}$ and

$$
I_{\alpha}=\exp \left(\frac{1}{16 \pi^{2}} \int_{\ln \Lambda_{\mathrm{FS}}}^{\ln \Lambda_{\mathrm{EW}}} \alpha \mathrm{d} t\right), \quad \Delta_{\tau}=\frac{C}{16 \pi^{2}} \int_{\ln \Lambda_{\mathrm{EW}}}^{\ln \Lambda_{\mathrm{FS}}} y_{\tau}^{2} \mathrm{~d} t .
$$

In the $\mathrm{SM}$ case, the RG running effect is negligible due to the smallness of $y_{\tau} \simeq 0.01$ (which renders $\left.\Delta_{\tau} \simeq \mathcal{O}\left(10^{-5}\right)\right)$. By contrast, $y_{\tau}^{2}=\left(1+\tan ^{2} \beta\right) m_{\tau}^{2} / v^{2}$ can be enhanced by a large $\tan \beta$ in the MSSM case. Given $\Lambda_{\mathrm{FS}} \simeq 10^{13} \mathrm{GeV}$, for example, the value of $\Delta_{\tau}$ depends on $\tan \beta$ in a way as

$$
\Delta_{\tau} \simeq 0.042\left(\frac{\tan \beta}{50}\right)^{2}
$$

With the help of eq. (2.31), one will get the RG-corrected neutrino mass matrix at $\Lambda_{\mathrm{EW}}$

$$
M_{\nu}\left(\Lambda_{\mathrm{EW}}\right) \simeq I_{\alpha}\left[M_{\nu}\left(\Lambda_{\mathrm{FS}}\right)-\Delta_{\tau}\left(\begin{array}{ccc}
0 & 0 & M_{e \tau} \\
0 & 0 & M_{\mu \tau} \\
M_{e \tau} & M_{\mu \tau} & 2 M_{\tau \tau}
\end{array}\right)\right]
$$

from a neutrino mass matrix respecting the $\mu-\tau$ reflection symmetry at $\Lambda_{\mathrm{FS}}$. By means of the above-mentioned treatment, one may arrange $M_{\nu}\left(\Lambda_{\mathrm{EW}}\right)$ in a form as given by eq. (2.6) with $\epsilon_{2}=2 \epsilon_{1}=\Delta_{\tau}$ and $\epsilon_{3,4}=0$. The relations between the mixing-parameter deviations and $\Delta_{\tau}$ can therefore be obtained by simply taking $\mathrm{R}_{2}=2 \mathrm{R}_{1}=\Delta_{\tau}$ and $\mathrm{I}_{1,2}=0$ in eq. (2.13). As before, the mixing-parameter deviations have a linear dependence on $\Delta_{\tau}$ and thus a square dependence on $\tan \beta$. In figures $6-7$ we display the mixing-parameter deviations respectively arising from $\tan \beta=50$ and $\tan \beta=30$ against the absolute neutrino mass scale for various combinations of $\rho^{(0)}$ and $\sigma^{(0)}$. By analogy with eq. (2.18) (with a square dependence in place of the linear dependence), the mixing-parameter deviations generated by other values of $\tan \beta$ can also be inferred from these results. Qualitatively, the mixing-parameter deviations produced by $\Delta_{\tau}$ closely resemble those resulting from $\mathrm{R}_{1,2}$ in a few aspects: (1) Their magnitudes tend to grow with the absolute neutrino mass 

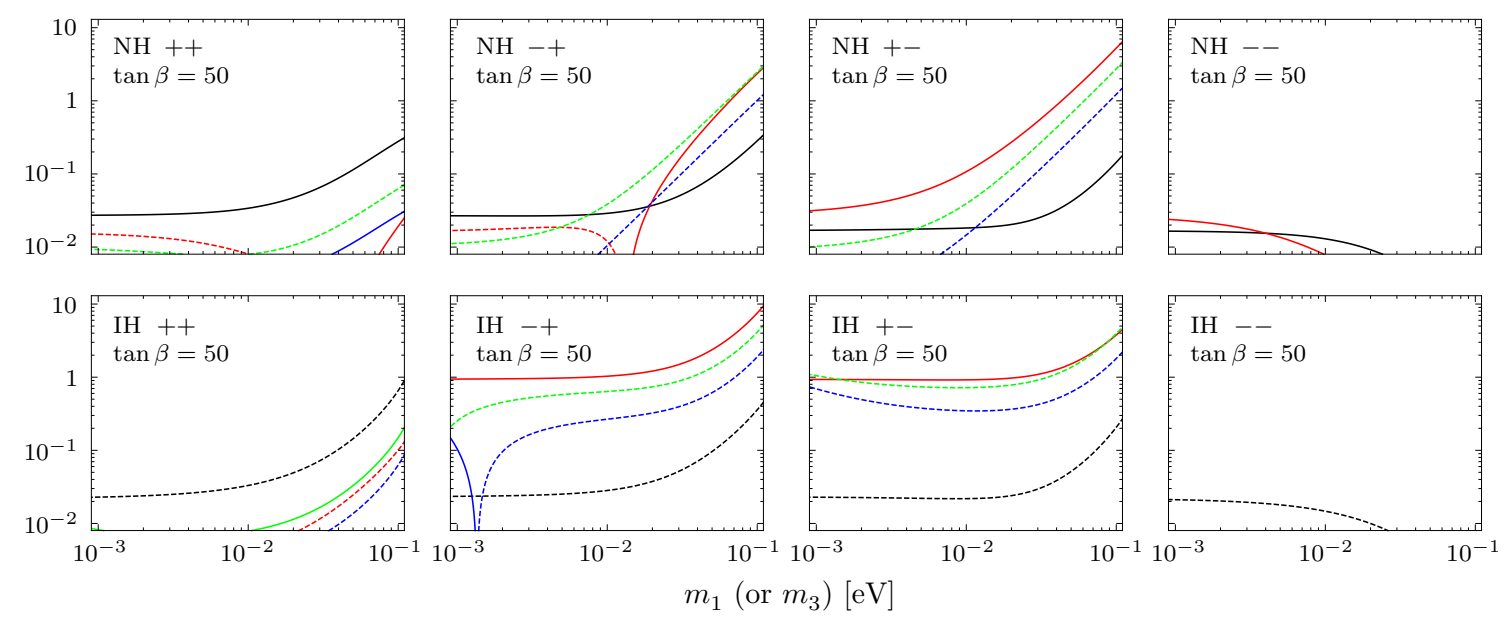

Figure 6. The mixing-parameter deviations in the MSSM case with $\tan \beta=50$ ( $\Delta \theta$ in black, $\Delta \delta$ in red, $\Delta \rho$ in green and $\Delta \sigma$ in blue) against the lightest neutrino mass $m_{1}$ (or $m_{3}$ ) in the NH (or $\mathrm{IH})$ case for various combinations of $\rho^{(0)}$ and $\sigma^{(0)}$ with $\delta^{(0)}=-\pi / 2$. The signs,,++-++- and - respectively stand for $\left[\rho^{(0)}, \sigma^{(0)}\right]=[0,0],[\pi / 2,0],[0, \pi / 2]$ and $[\pi / 2, \pi / 2]$.
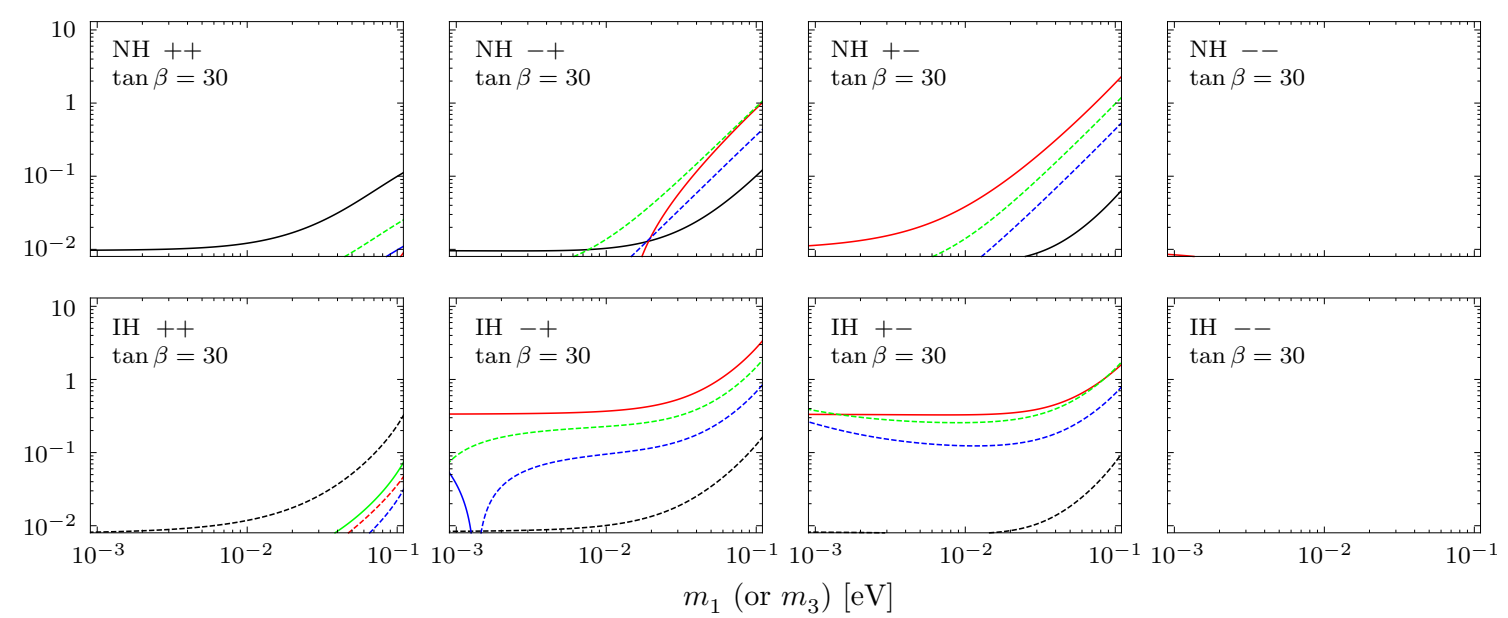

Figure 7. The mixing-parameter deviations in the MSSM case with $\tan \beta=30$ ( $\Delta \theta$ in black, $\Delta \delta$ in red, $\Delta \rho$ in green and $\Delta \sigma$ in blue) against the lightest neutrino mass $m_{1}$ (or $m_{3}$ ) in the $\mathrm{NH}$ (or $\mathrm{IH})$ case for various combinations of $\rho^{(0)}$ and $\sigma^{(0)}$ with $\delta^{(0)}=-\pi / 2$. The signs,,++-++- and -- respectively stand for $\left[\rho^{(0)}, \sigma^{(0)}\right]=[0,0],[\pi / 2,0],[0, \pi / 2]$ and $[\pi / 2, \pi / 2]$.

scale (except in the case of $\left[\rho^{(0)}, \sigma^{(0)}\right]=[\pi / 2, \pi / 2]$ ). (2) $\Delta \theta$ generally receives a modest contribution from $\Delta_{\tau}$ in most cases, while $\Delta \delta, \Delta \rho$ and $\Delta \sigma$ may get remarkably magnified for $\rho^{(0)} \neq \sigma^{(0)}$. (3) $|\Delta \delta|$ and $|\Delta \rho|$ are comparable to each other, while $|\Delta \sigma|$ is somewhat smaller. (4) $\Delta \theta$ is always positive (negative) in the NH (IH) case. Quantitatively, the allowed $\Delta \theta$ and $\Delta \delta$ from $\Delta_{\tau} \leq 0.04$ (for $\tan \beta \leq 50$ ) are as follows: (1) In the case of $m_{1} \ll m_{2} \ll m_{3}$ or $m_{3} \ll m_{1} \simeq m_{2},|\Delta \theta|$ is smaller than 0.02 . When the neutrino masses are quasi-degenerate (except in the case of $\left.\left[\rho^{(0)}, \sigma^{(0)}\right]=[\pi / 2, \pi / 2]\right),|\Delta \theta|$ may receive a contribution up to 0.3 , implying that $\Delta_{\tau} \simeq 0.02$ (corresponding to $\tan \beta \simeq 35$ ) is large 
enough to generate $|\Delta \theta| \simeq 0.1$. (2) $|\Delta \delta|$ is also smaller than 0.02 for $m_{1} \ll m_{2} \ll m_{3}$. In the case of $m_{3} \ll m_{1} \simeq m_{2}$ or $m_{1} \simeq m_{2} \simeq m_{3},|\Delta \delta|$ will be greatly enhanced to $\mathcal{O}(1)$ for $\rho^{(0)} \neq \sigma^{(0)}$ (even $\Delta_{\tau}$ of $\mathcal{O}(0.001)$ that corresponds to $\tan \beta \simeq 10$ is sufficient for producing $|\Delta \delta|$ of $\mathcal{O}(0.1))$ but negligibly small for $\left[\rho^{(0)}, \sigma^{(0)}\right]=[\pi / 2, \pi / 2]$.

On the other hand, the analytical expressions for $\Delta \theta, \Delta \rho$ and $\Delta$ are found to be

$$
\begin{aligned}
\Delta \theta \simeq & {\left[2\left(\bar{m}_{1}-m_{3}\right)\left(\bar{m}_{2}-m_{3}\right)\right]^{-1}\left[m_{12}^{2}-\left(m_{11}-m_{3}\right)\left(m_{22}+m_{3}\right)\right] \Delta_{\tau}, } \\
\Delta \delta \simeq & -\left[\mathrm{T}\left(\bar{m}_{1}+\bar{m}_{2}\right)\left(\bar{m}_{1}-m_{3}\right)\left(\bar{m}_{2}-m_{3}\right) \bar{s}_{13}\right]^{-1}\left[\mathrm{~T} m_{12} m_{3}\left(\bar{m}_{1}+\bar{m}_{2}-2 m_{3} s_{13}^{2}\right)\right. \\
& \left.-2\left(m_{11}+\mathrm{T} m_{12}+m_{3}\right)\left(\bar{m}_{1} \bar{m}_{2}-m_{22} m_{3}\right) s_{13}^{2}\right] \Delta_{\tau}, \\
\Delta \rho \simeq & -\left[2 \bar{m}_{1} m_{3}\left(\bar{m}_{1}+\bar{m}_{2}\right)\left(\bar{m}_{1}-m_{3}\right)\left(\bar{m}_{2}-m_{3}\right) \bar{s}_{13} t_{12}\right]^{-1}\left\{\mathrm { T } m _ { 1 2 } m _ { 3 } ^ { 2 } ( \overline { m } _ { 1 } + \overline { m } _ { 2 } ) \left(m_{11}\right.\right. \\
& \left.-m_{3} s_{13}^{2}+2 m_{22} t_{12}^{2} s_{13}^{2}\right)-m_{11}\left[\mathrm{~T} m_{12} m_{3}\left(\bar{m}_{1}+\bar{m}_{2}-2 m_{3} s_{13}^{2}\right)-2\left(m_{11}+\mathrm{T} m_{12}\right.\right. \\
& \left.\left.\left.+m_{3}\right)\left(\bar{m}_{1} \bar{m}_{2}-m_{22} m_{3}\right) s_{13}^{2}\right]\left[m_{3}+m_{11} \Omega s_{13}^{2}-m_{22} t_{12}^{2} s_{13}^{2}\right]\right\} \Delta_{\tau},
\end{aligned}
$$

with $t_{12}=\tan \theta_{12}$, while $\Delta \sigma$ can be obtained from $\bar{m}_{1} \Delta \rho / \bar{m}_{2}$ by making the replacement $t_{12} \rightarrow-1 / t_{12}$. These results can help us understand the numerical results: (1) For $m_{1} \ll$ $m_{2} \ll m_{3}$, eq. (2.35) is simplified to

$$
\Delta \theta \simeq \frac{1}{2} \Delta_{\tau}, \quad \Delta \delta \simeq \frac{\bar{m}_{2} c_{12} s_{12}}{m_{3} \bar{s}_{13}} \Delta_{\tau}, \quad \Delta \rho \simeq \frac{c_{12} \bar{s}_{13}}{s_{12}} \Delta_{\tau} .
$$

(2) In the case of $m_{1} \simeq m_{2} \gg m_{3}$, one will have

$$
\Delta \delta \simeq \frac{1}{\mathrm{~T}} \bar{s}_{13} \Delta_{\tau}, \quad \Delta \rho \simeq-\frac{\left[m_{3}+\bar{m}_{1}\left(1-t_{12}^{2}\right) s_{13}^{2}\right] \bar{s}_{13}}{2 m_{3} t_{12}} \Delta_{\tau}
$$

for $\rho^{(0)}=\sigma^{(0)}$, or

$$
\Delta \delta \simeq-\frac{8}{|r|} c_{12} s_{12} \bar{s}_{13} \Delta_{\tau}, \quad \Delta \rho \simeq \frac{8\left[m_{3}+\bar{m}_{1}\left(c_{12}^{2}-s_{12}^{2}\right)\left(1+t_{12}^{2}\right) s_{13}^{2}\right] c_{12}^{3} s_{12} \bar{s}_{13}}{m_{3}|r|} \Delta_{\tau},
$$

for $\rho^{(0)} \neq \sigma^{(0)}$ together with $\Delta \theta \simeq-1 / 2 \Delta_{\tau}$. (3) When the case of $m_{1} \simeq m_{2} \simeq m_{3} \simeq m_{0}$ is considered, the results become

$$
\begin{aligned}
& \Delta \theta \simeq \frac{2 m_{0}^{2}}{\Delta m_{31}^{2}} \Delta_{\tau}, \quad \Delta \delta \simeq \frac{2 m_{0}^{2}\left[\mathrm{Trc}_{12} s_{12}-2 s_{13}^{2}\right]}{\mathrm{T} \Delta m_{31}^{2} \bar{s}_{13}} \Delta_{\tau}, \quad \Delta \rho \simeq \frac{\mathrm{T}_{0}^{2} r c_{12}^{2}}{\Delta m_{31}^{2} \bar{s}_{13}} \Delta_{\tau} ; \\
& \Delta \theta \simeq \frac{2 m_{0}^{2} c_{12}^{2}}{\Delta m_{31}^{2}} \Delta_{\tau}, \quad \Delta \delta \simeq \frac{2 m_{0}^{2}\left(r-4 s_{13}^{2}\right) c_{12} s_{12}}{\Delta m_{21}^{2} \bar{s}_{13}} \Delta_{\tau}, \quad \Delta \rho \simeq \frac{8 m_{0}^{2} c_{12}^{3} s_{12} \bar{s}_{13}}{\Delta m_{21}^{2}} \Delta_{\tau} ; \\
& \Delta \theta \simeq \frac{2 m_{0}^{2} s_{12}^{2}}{\Delta m_{31}^{2}} \Delta_{\tau}, \quad \Delta \delta \simeq-\frac{2 m_{0}^{2}\left(r+4 s_{13}^{2}\right) c_{12} s_{12}}{\Delta m_{21}^{2} \bar{s}_{13}} \Delta_{\tau}, \quad \Delta \rho \simeq \frac{8 m_{0}^{2} c_{12}^{3} s_{12} \bar{s}_{13}}{\Delta m_{21}^{2}} \Delta_{\tau} ; \\
& \Delta \theta \simeq \frac{\Delta m_{31}^{2}}{8 m_{0}^{2}} \Delta_{\tau}, \quad \Delta \delta \simeq-\frac{\mathrm{T} \Delta m_{21}^{2} c_{12} s_{12}+2 \Delta m_{31}^{2} s_{13}^{2}}{8 \mathrm{~T} m_{0}^{2} \bar{s}_{13}} \Delta_{\tau}, \quad \Delta \rho \simeq \frac{\Delta m_{31}^{2} \bar{s}_{13}}{8 m_{0}^{2} t_{12}} \Delta_{\tau},
\end{aligned}
$$

for $\left[\rho^{(0)}, \sigma^{(0)}\right]=[0,0],[\pi / 2,0],[0, \pi / 2]$ and $[\pi / 2, \pi / 2]$. One can see that these approximation results agree well with the corresponding numerical results. 


\section{Summary}

To summarize, the $\mu-\tau$ reflection symmetry deserves particular attention as it leads to the interesting results $\theta_{23}=\pi / 4$ and $\delta= \pm \pi / 2$ (which are close to the current experimental data) as well as trivial Majorana phases. Nevertheless, it is reasonable for us to consider the breaking of such a symmetry either from the theoretical considerations (e.g., the RG running effect may provide a source for the symmetry breaking) or on the basis of experimental results (e.g., the newly-reported NOvA result disfavors the maximal mixing scenario at a $2.6 \sigma$ level). Consequently, we have performed a systematic study for the possible symmetry-breaking patterns and their implications for the mixing parameters.

We first define some parameters measuring the symmetry-breaking strengths and then derive an equation set relating them with the deviations of mixing parameters from the special values taken in the symmetry context. By solving these equations in both a numerical and analytical way, the sensitivity of mixing-parameter deviations to the symmetrybreaking parameters for various neutrino mass schemes and the Majorana-phase combinations are investigated in some detail. It turns out that $\Delta \theta$ is most sensitive to $\mathrm{R}_{2}$ while $\Delta \delta$, $\Delta \rho$ and $\Delta \sigma$ to all the symmetry-breaking parameters. And the magnitudes of $\Delta \delta, \Delta \rho$ and $\Delta \sigma$ are generally much greater than that of $\Delta \theta$. This means that the symmetry-breaking pattern with a sizable $\mathrm{R}_{2}$ will be favored if $\Delta \theta$ is notable, but any symmetry-breaking pattern may cause significant $\Delta \delta, \Delta \rho$ and $\Delta \sigma$. The mixing-parameter deviations tend to get remarkably magnified (a symmetry-breaking parameter of $\leq \mathcal{O}(0.01)$ may give rise to some mixing-parameter deviations of $\geq \mathcal{O}(0.1))$ in the case of $m_{1} \simeq m_{2} \simeq m_{3}$ combined with $\rho^{(0)} \neq \sigma^{(0)}$. But they will be highly suppressed and inconsiderable in the case of $m_{1} \simeq m_{2} \simeq m_{3}$ combined with $\left[\rho^{(0)}, \sigma^{(0)}\right]=[\pi / 2, \pi / 2]$. With these general results as guide, one may easily find an appropriate specific way to break the $\mu-\tau$ reflection symmetry so as to generate the required mixing-parameter deviations when necessary. Finally, as a unique illustration, the general treatment is applied to the specific symmetry breaking induced by the RG running effect.

\section{Acknowledgments}

I would like to thank Professor Zhi-zhong Xing for fruitful collaboration on the $\mu-\tau$ flavor symmetry. This work is supported in part by the National Natural Science Foundation of China under grant No. 11605081.

Open Access. This article is distributed under the terms of the Creative Commons Attribution License (CC-BY 4.0), which permits any use, distribution and reproduction in any medium, provided the original author(s) and source are credited.

\section{References}

[1] Particle Data Group collaboration, C. Patrignani et al., Review of Particle Physics, Chin. Phys. C 40 (2016) 100001 [InSPIRE].

[2] F. Capozzi, G.L. Fogli, E. Lisi, A. Marrone, D. Montanino and A. Palazzo, Status of three-neutrino oscillation parameters, circa 2013, Phys. Rev. D 89 (2014) 093018 [arXiv:1312.2878] [INSPIRE]. 
[3] Planck collaboration, P.A.R. Ade et al., Planck 2015 results. XIII. Cosmological parameters, Astron. Astrophys. 594 (2016) A13 [arXiv: 1502.01589] [INSPIRE].

[4] NOvA collaboration, P. Adamson et al., Measurement of the neutrino mixing angle $\theta_{23}$ in NOvA, Phys. Rev. Lett. 118 (2017) 151802 [arXiv:1701.05891] [INSPIRE].

[5] I. Esteban, M.C. Gonzalez-Garcia, M. Maltoni, I. Martinez-Soler and T. Schwetz, Updated fit to three neutrino mixing: exploring the accelerator-reactor complementarity, JHEP 01 (2017) 087 [arXiv: 1611.01514] [INSPIRE].

[6] G. Altarelli and F. Feruglio, Discrete Flavor Symmetries and Models of Neutrino Mixing, Rev. Mod. Phys. 82 (2010) 2701 [arXiv:1002.0211] [InSPIRE].

[7] S.F. King and C. Luhn, Neutrino Mass and Mixing with Discrete Symmetry, Rept. Prog. Phys. 76 (2013) 056201 [arXiv: 1301.1340] [INSPIRE].

[8] T. Fukuyama and H. Nishiura, Mass matrix of Majorana neutrinos, hep-ph/9702253 [INSPIRE].

[9] E. Ma and M. Raidal, Neutrino mass, muon anomalous magnetic moment and lepton flavor nonconservation, Phys. Rev. Lett. 87 (2001) 011802 [Erratum ibid. 87 (2001) 159901] [hep-ph/0102255] [INSPIRE].

[10] C.S. Lam, A 2-3 symmetry in neutrino oscillations, Phys. Lett. B 507 (2001) 214 [hep-ph/0104116] [INSPIRE].

[11] K.R.S. Balaji, W. Grimus and T. Schwetz, The Solar LMA neutrino oscillation solution in the Zee model, Phys. Lett. B 508 (2001) 301 [hep-ph/0104035] [INSPIRE].

[12] Z.-z. Xing and Z.-h. Zhao, A review of $\mu-\tau$ flavor symmetry in neutrino physics, Rept. Prog. Phys. 79 (2016) 076201 [arXiv: 1512.04207] [INSPIRE].

[13] CHOOZ collaboration, M. Apollonio et al., Initial results from the CHOOZ long baseline reactor neutrino oscillation experiment, Phys. Lett. B 420 (1998) 397 [hep-ex/9711002] [INSPIRE].

[14] DAYA BAy collaboration, F.P. An et al., Observation of electron-antineutrino disappearance at Daya Bay, Phys. Rev. Lett. 108 (2012) 171803 [arXiv:1203.1669] [InSPIRE].

[15] S. Gupta, A.S. Joshipura and K.M. Patel, How good is $\mu-\tau$ symmetry after results on non-zero $\theta_{13}$ ?, JHEP 09 (2013) 035 [arXiv: 1301.7130] [INSPIRE].

[16] P.F. Harrison and W.G. Scott, mu - tau reflection symmetry in lepton mixing and neutrino oscillations, Phys. Lett. B 547 (2002) 219 [hep-ph/0210197] [INSPIRE].

[17] F. Feruglio, C. Hagedorn and R. Ziegler, Lepton Mixing Parameters from Discrete and CP Symmetries, JHEP 07 (2013) 027 [arXiv: 1211.5560] [INSPIRE].

[18] M. Holthausen, M. Lindner and M.A. Schmidt, CP and Discrete Flavour Symmetries, JHEP 04 (2013) 122 [arXiv:1211.6953] [INSPIRE].

[19] W. Grimus and L. Lavoura, A Nonstandard CP transformation leading to maximal atmospheric neutrino mixing, Phys. Lett. B 579 (2004) 113 [hep-ph/0305309] [INSPIRE].

[20] P.M. Ferreira, W. Grimus, L. Lavoura and P.O. Ludl, Maximal CP-violation in Lepton Mixing from a Model with Delta(27) flavour Symmetry, JHEP 09 (2012) 128 [arXiv: 1206.7072] [INSPIRE].

[21] W. Grimus and L. Lavoura, mu-tau Interchange symmetry and lepton mixing, Fortsch. Phys. 61 (2013) 535 [arXiv: 1207.1678] [INSPIRE]. 
[22] R.N. Mohapatra and C.C. Nishi, $S_{4}$ Flavored CP Symmetry for Neutrinos, Phys. Rev. D 86 (2012) 073007 [arXiv: 1208.2875] [INSPIRE].

[23] R.N. Mohapatra and C.C. Nishi, Implications of $\mu$ - $\tau$ flavored CP symmetry of leptons, JHEP 08 (2015) 092 [arXiv: 1506.06788] [INSPIRE].

[24] E. Ma, A. Natale and O. Popov, Neutrino Mixing and CP Phase Correlations, Phys. Lett. B 746 (2015) 114 [arXiv:1502.08023] [INSPIRE].

[25] E. Ma, Transformative $A_{4}$ mixing of neutrinos with CP-violation, Phys. Rev. D 92 (2015) 051301 [arXiv: 1504.02086] [INSPIRE].

[26] E. Ma, Neutrino mixing: $A_{4}$ variations, Phys. Lett. B 752 (2016) 198 [arXiv:1510.02501] [INSPIRE].

[27] A.S. Joshipura and K.M. Patel, Generalized $\mu-\tau$ symmetry and discrete subgroups of $O(3)$, Phys. Lett. B 749 (2015) 159 [arXiv:1507.01235] [INSPIRE].

[28] H.-J. He, W. Rodejohann and X.-J. Xu, Origin of Constrained Maximal CP-violation in Flavor Symmetry, Phys. Lett. B 751 (2015) 586 [arXiv:1507.03541] [INSPIRE].

[29] C.C. Nishi, New and trivial CP symmetry for extended $A_{4}$ flavor, Phys. Rev. D 93 (2016) 093009 [arXiv: 1601.00977] [INSPIRE].

[30] P.M. Ferreira, W. Grimus, D. Jurciukonis and L. Lavoura, Scotogenic model for co-bimaximal mixing, JHEP 07 (2016) 010 [arXiv: 1604.07777] [INSPIRE].

[31] A.S. Joshipura and N. Nath, Neutrino masses and mixing in $A_{5}$ with flavor antisymmetry, Phys. Rev. D 94 (2016) 036008 [arXiv:1606.01697] [INSPIRE].

[32] C.-C. Li, J.-N. Lu and G.-J. Ding, $A_{4}$ and CP symmetry and a model with maximal CP-violation, Nucl. Phys. B 913 (2016) 110 [arXiv:1608.01860] [InSPIRE].

[33] C.C. Nishi and B.L. Sánchez-Vega, Mu-tau reflection symmetry with a texture-zero, JHEP 01 (2017) 068 [arXiv: 1611.08282] [INSPIRE].

[34] W. Rodejohann and X.-J. Xu, Trimaximal $\mu-\tau$ reflection symmetry, arXiv:1705.02027 [INSPIRE].

[35] W. Grimus, A.S. Joshipura, S. Kaneko, L. Lavoura, H. Sawanaka and M. Tanimoto, Non-vanishing U(e3) and cos 2 theta(23) from a broken Z(2) symmetry, Nucl. Phys. B 713 (2005) 151 [hep-ph/0408123] [INSPIRE].

[36] W. Rodejohann, Neutrino-less Double Beta Decay and Particle Physics, Int. J. Mod. Phys. E 20 (2011) 1833 [arXiv:1106.1334] [INSPIRE].

[37] S.M. Bilenky and C. Giunti, Neutrinoless Double-Beta Decay: a Probe of Physics Beyond the Standard Model, Int. J. Mod. Phys. A 30 (2015) 1530001 [arXiv:1411.4791] [InSPIRE].

[38] S. Dell'Oro, S. Marcocci, M. Viel and F. Vissani, Neutrinoless double beta decay: 2015 review, Adv. High Energy Phys. 2016 (2016) 2162659 [arXiv:1601.07512] [INSPIRE].

[39] J.D. Vergados, H. Ejiri and F. Šimkovic, Neutrinoless double beta decay and neutrino mass, Int. J. Mod. Phys. E 25 (2016) 1630007 [arXiv:1612.02924] [INSPIRE].

[40] T. Ohlsson and S. Zhou, Renormalization group running of neutrino parameters, Nature Commun. 5 (2014) 5153 [arXiv:1311.3846] [INSPIRE].

[41] Y.-L. Zhou, $\mu-\tau$ reflection symmetry and radiative corrections, arXiv:1409.8600 [INSPIRE]. 
[42] Y.H. Ahn, S.K. Kang, C.S. Kim and T.P. Nguyen, Bridges of Low Energy observables with Leptogenesis in mu-tau Reflection Symmetry, arXiv:0811.1458 [INSPIRE].

[43] S. Luo and Z.-z. Xing, Resolving the octant of $\theta_{23}$ via radiative $\mu-\tau$ symmetry breaking, Phys. Rev. D 90 (2014) 073005 [arXiv:1408.5005] [INSPIRE].

[44] J. Zhang and S. Zhou, Viability of exact tri-bimaximal, golden-ratio and bimaximal mixing patterns and renormalization-group running effects, JHEP 09 (2016) 167 [arXiv: 1606.09591] [INSPIRE].

[45] P.H. Chankowski and Z. Pluciennik, Renormalization group equations for seesaw neutrino masses, Phys. Lett. B 316 (1993) 312 [hep-ph/9306333] [INSPIRE].

[46] K.S. Babu, C.N. Leung and J.T. Pantaleone, Renormalization of the neutrino mass operator, Phys. Lett. B 319 (1993) 191 [hep-ph/9309223] [INSPIRE].

[47] S. Antusch, M. Drees, J. Kersten, M. Lindner and M. Ratz, Neutrino mass operator renormalization revisited, Phys. Lett. B 519 (2001) 238 [hep-ph/0108005] [INSPIRE].

[48] S. Antusch, M. Drees, J. Kersten, M. Lindner and M. Ratz, Neutrino mass operator renormalization in two Higgs doublet models and the MSSM, Phys. Lett. B 525 (2002) 130 [hep-ph/0110366] [INSPIRE].

[49] S. Antusch, J. Kersten, M. Lindner and M. Ratz, Running neutrino masses, mixings and CP phases: Analytical results and phenomenological consequences, Nucl. Phys. B 674 (2003) 401 [hep-ph/0305273] [INSPIRE].

[50] J.R. Ellis and S. Lola, Can neutrinos be degenerate in mass?, Phys. Lett. B 458 (1999) 310 [hep-ph/9904279] [INSPIRE].

[51] P.H. Chankowski, W. Krolikowski and S. Pokorski, Fixed points in the evolution of neutrino mixings, Phys. Lett. B 473 (2000) 109 [hep-ph/9910231] [INSPIRE]. 